\title{
Transfer learning for Turkish named entity recognition on noisy text
}

\author{
EMRE KA ĞAN AKKAYA, BURCU CAN \\ Hacettepe Üniversity, Turkey
}

( Received 26 October 2018)

\begin{abstract}
In this article, we investigate using deep neural networks with different word representation techniques for named entity recognition (NER) on Turkish noisy text. We argue that valuable latent features for NER can, in fact, be learned without using any hand-crafted features and/or domain-specific resources such as gazetteers and lexicons. In this regard, we utilize character level, character n-gram level, morpheme-level, and orthographic character-level word representations. Since noisy data with NER annotation is scarce for Turkish, we introduce a transfer learning model in order to learn infrequent entity types as an extension to the Bi-LSTM-CRF architecture by incorporating an additional CRF layer that is trained on a larger (but formal) text and a noisy text simultaneously. This allows us to learn from both formal and informal/noisy text, thus, improve the performance of our model further for rarely-seen entity types. We experimented on Turkish as a morphologically-rich language and English as a relatively morphologically-poor language. We obtained an entity-level F1 score of $67.39 \%$ on Turkish noisy data and $45.30 \%$ on English noisy data, which outperforms the current state-of-art models on noisy text. The English scores are lower compared to Turkish scores because of the intense sparsity in the data introduced by the user writing styles. The results prove that using subword information significantly contributes to learning latent features for morphologically-rich languages.
\end{abstract}

Named entity recognition; Transfer learning; Recurrent neural networks; Lowresource language; Noisy text

\section{Introduction}

Named Entity Recognition (NER) is an information extraction task in Natural Language Processing (NLP) that aims to identify and categorize each word into pre-defined categories. For example, for the sentence "Thomas Bayes was the son of London Presbyterian minister Joshua Bayes.", as a named entity recognition task we aim to assign PERSON label for Thomas Bayes and Joshua Bayes, and assign LOCATION label for London, and CORPORATION label for Presbyterian. As we can see from the recent studies in the literature, the performance of named entity recognition on formal (e.g. newspapers, academic papers) data is very high, particularly for languages like English with comparably poor morphology and abundant 
annotated data. Recent research such as Lample et al. (2016) and Ma and Hovy (2016) achieved over 91\% F1 score on English formal data, almost comparable to human annotation performance. So one can prematurely conclude that the NER task has nearly reached its peak performance.

However with the ever-changing nature of the Internet, especially after the emergence of social media, we have been introduced to informal/noisy data (usergenerated data) such as user comments and tweets. This new type of data is highly valuable for information extraction tasks such as opinion mining due to being widespread and having almost up-to-date nature. However, noisy and informal text normally includes missing characters in words (either deliberately or by forgetfulness), missing punctuation, various emojis, slang words and abbreviations.

All of these bring new problems for the existing Turkish NER studies, considering most of them are either statistical or rule-based models that usually depend on manually-crafted features (e.g. capitalization, numerical/date/time patterns or other rule-based features) and/or external domain-specific resources (e.g. gazetteers, lexicons), therefore ill-suited to noisy data. Some of these studies try to solve these new challenges either by extending their existing feature set to better suit on this new domain or by adding new domain-specific resources. Current state-ofthe-art Turkish NER model (Şeker and Eryiğit, 2017) which is a CRF-based model utilizing domain-specific heavy feature engineering and external resources achieves an F1 score of $91.94 \%$ on formal data and only $67.96 \%$ (and $63.63 \%$ without using Twitter mention feature to label mentions as PERSON) on noisy data. This is mostly due to Turkish being a morphologically-rich and agglutinative language and having scarce annotated data. It is evident that Turkish NER performance is far behind on noisy/informal data, despite being a well-studied topic for formal data. Recent successful studies on other languages, especially on English, address these issues by utilizing neural architectures with the help of auto-generated features.

Turkish being a morphologically complex language, Named Entity Recognition receive's its own share. Although derivation in Turkish named entities is not very common, inflected named entities are seen quite often in Turkish. Especially case markers are seen with any type of named entity. For example, in the sentence "Istanbul'a gideceğim" (means I will go to Istanbul."), the location named entity 'Istanbul' is inflected in the dative case; "Onu Ahmet'ten alabilirsin." (means "You can take it from Ahmet."), the person name 'Ahmet' is inflected in ablative case. Thereby due to the common usage of su xes with named entities, the sparsity problem is introduced in NER task. The problem is even more severe in noisy text, since the morphemes also could be spelled differently by different users on social media. For example, the su x 'ciğim' that means 'dear' when it is used with person names, could be written as 'cim', or as 'çim', 'çum', 'çım', 'cım', 'cim' etc., depending on the last vowel in the name accordingly with the vowel harmony.

This motivates us to research and adopt deep recurrent neural network models for Turkish and obtain valuable features without using any external domain-specific resources or any hand-crafted features. To this end, we propose a transfer learning model that is an extension of the widely used bidirectional LSTM-CRF model by incorporating an additional CRF layer that we train on another, preferably larger 
dataset to overcome the annotated data scarcity problem. The model is rather similar to the model presented by von Däniken and Cieliebak (2017) since we also use another CRF layer to further improve the performance. We argue that subword information is crucial in word representation for morphologically-rich and agglutinative languages therefore the model also utilizes different subword embeddings such as morph2vec (Üstün et al., 2018), fasttext (Bojanowski et al., 2017) and orthographic character-level embeddings. Morph2vec (Üstün et al., 2018) is a word representation model that estimates the word embeddings through its morphemes where the segmentation of words are not required a priori, therefore the pre-trained word embeddings are mimicked by using an attention mechanism over a list of potential segmentations of each word to obtain the final word representation. On the contrary, fasttext (Bojanowski et al., 2017) estimates the word embeddings through the n-grams of each word. To the best of our knowledge, this is the first neural network model without using any hand-crafted features and external resources for Turkish named entity recognition. Consequently, we obtain an F1 score of $67.39 \%$ on Turkish noisy data and $45.30 \%$ on English noisy data, which are both the highest scores for both languages.

The paper is organized as follows: Section 2 reviews the recent work on named entity recognition on noisy text for Turkish and also for English, section 3 describes the word representation methods used for representing each word by a dense vector in the named entity recognition models proposed in this paper, section 4 describes and gives the mathematical definition of the baseline Bi-LSTM-CRF model (Huang et al., 2015) adopted in this article, section 5 describes the proposed transfer learning model, section 6 gives the details on datasets and on the implementation of the models in addition to the experimental results obtained from the proposed models on Turkish and English, and finally section 7 concludes the paper along with the future goals.

\section{Related Work}

Various methods have been adopted for named entity recognition, those include statistical methods (Bikel et al., 1997; Wu et al., 2003; Suzuki and Isozaki, 2008), rule-based models (Petasis et al., 2001), and recently deep neural network architectures (Huang et al., 2015; Ma and Hovy, 2016). Since user-generated text on the Internet is typically different compared to formal text, more latent features need to be defined manually or more sophisticated methods need to be used to learn the latent features automatically from a given text. This is because noisy text is more scarce compared to formal text since it may change from one user to another.

One of the commonly used features would be the meaning of the words. Although the spelling of each word may change from one text to another, the meaning would stay the same. Meaning representation have benefited from distributional approaches a lot. In recent years, distributional models such as Latent Semantic Analysis (LSA) (Landauer et al., 1998) have changed direction towards neural models. Word representation models such as word2vec (Mikolov et al., 2013) or GloVe (Pennington et al., 2014) have shown superior performance. However, those models 
learn word representations very well when there is enough contextual information for each word, which will not be true for the scarce and noisy text. Therefore, other neural models that make use of subword information such as characters (Cao and Rei, 2016), character n-grams (Bojanowski et al., 2017), and morphemes (Üstün et al., 2018) have been introduced, which learn the representations of scarce data (i.e., noisy text or any text in a morphologically-rich language) better than wordlevel models. Neural word embeddings obtained from such models have been used as features and have shown superior performance when they are sequentially encoded by LSTMs. Moreover, it has been discovered that an additional CRF layer can learn the named entities by using the latent features learned by the LSTMs, which introduces the well-known Bi-LSTM-CRF (bidirectional Long Short Term Memory and Conditional Random Field) architecture (Huang et al., 2015) as a sequence labeling model.

Here, we review mainly the research on named entity recognition for noisy text. Although the main scope of this article is Turkish NER, since we are also inspired by other models on English, we also review the research on English named entity recognition on noisy text.

\subsection{Named Entity Recognition on Turkish Noisy Data}

First of all, it is worth mentioning that all studies reported in this section use the same Turkish noisy dataset, so the reported scores are comparable to each other ${ }^{1}$.

Çelikkaya et al. (2013) introduce the first study focusing on noisy data for Turkish with a CRF-based model that utilizes hand-crafted morphological and lexical features (e.g. stem, PoS tag, noun case, lower/upper case) along with gazetteers. They reported an F1 score of $19.28 \%$ on noisy dataset and $91.64 \%$ on formal dataset which can be interpreted as another indication that NER on noisy data does not perform as well as NER on formal text. With the aim of adapting the model for noisy data, Küçük and Steinberger (2014) extend a previous multilingual rule-based NER system by expanding existing domain-specific resources based on the fact that most sentences in the noisy data misses the letters with diacritics (ç, ğ, $, ~, \ddot{o}, s, ~ \ddot{u})$ and the authors employ a normalization scheme using this feature. As a result, they achieved an F1 score of $46.93 \%$ on the same Turkish noisy dataset.

Eken and Tantuğ (2015) introduce another CRF-based approach that also makes use of gazetteers (with optional distance-based matching) and numerous features (e.g. apostrophe character, case of the word, start of sentence) along with the word su xes and prefixes. They reported $46.97 \%$ F1 score on a new noisy imbalanced dataset, and $28.53 \%$ F1 score on the same Turkish noisy dataset. Okur et al. (2016) present a regularized averaged multiclass perceptron with hand-crafted features (e.g. word type flags, su $\mathrm{x}$, prefix, capitalization) along with pre-trained embeddings obtained from word2vec (Mikolov et al., 2013). They also perform tweet normalization using the model introduced by Torunoğlu and Eryiğit (2014). Consequently, they obtain an F1 score of $48.96 \%$ on the noisy dataset.

${ }^{1}$ The details of the noisy dataset are given in Section 6.3. 
Şeker and Eryiğit (2017) present the state-of-the-art model on Turkish NER which is another CRF-based model, similar to that of Çelikkaya et al. (2013). The authors use an extensive set of morphological and lexical features (e.g. stem, part-ofspeech tags, capitalization, word type and shape flags) and gazetteers. Additionally, they use the existence of Twitter mentions as a feature. They also provide the reannotated versions of the two commonly used Turkish datasets: news dataset (Tür et al., 2003) and Twitter dataset (Çelikkaya et al., 2013). Re-annotated versions also include TIMEX and NUMEX types along with previously-labeled ENAMEX types. Finally, they report an F1 score of $67.96 \%$ with Twitter mentions and $63.63 \%$ without the mentions on the re-annotated version of the Turkish noisy dataset.

\subsection{Named Entity Recognition on English Noisy Data}

Analogously, all studies reported in this section use the same English noisy dataset, which was provided by the 3rd Workshop on Noisy User-Generated Text at EMNLP (WNUT'2017) ${ }^{2}$ so that all the reported results are comparable to each other.

Aguilar et al. (2017), the winner of the WNUT' $17,{ }^{3}$ apply multi-task learning approach with a CRF-based model that incorporates pre-trained word embeddings obtained from word2vec (Mikolov et al., 2013) and orthographic character-level embeddings trained on a CNN with 2-stacked convolutional layers. They also make use of gazetteers for the well-known entities. They report an F1 score of $41.86 \%$ on entity-level and $40.24 \%$ on surface forms.

von Däniken and Cieliebak (2017) use a transfer learning model. One of our proposed models is also based on their model. However, unlike our model their model incorporates sentence-level embeddings (sent2vec) (Pagliardini et al., 2017) and capitalization features in addition to character-level embeddings trained by a CNN and pre-trained word embeddings obtained from fasttext (Bojanowski et al., 2017). As a result, they obtain $40.78 \% \mathrm{~F} 1$ score on entity-level and $39.33 \% \mathrm{~F} 1$ score on surface forms. Lin et al. (2017) follow a similar approach for a CRF-based model and use word embeddings that are obtained from pre-trained word embeddings and character-level embeddings obtained from another bidirectional LSTM. They also incorporate syntactic information by using part-of-speech (POS) tags, dependency roles, and word position, and head position. They achieve an F1 score of $40.42 \%$ on entity-level and $37.62 \%$ on surface-forms.

Sikdar and Gambäck (2017) propose an ensemble-based approach that uses features learned from CRF, support vector machine (SVM) and an LSTM. They also use hand-crafted features such as PoS tags, local context, chunk, su $\mathrm{x}$ and prefix, word frequency and a collection of flags (e.g. is-word-length-less-than-5, is-all-digit etc.). Consequently, they achieve $38.35 \%$ F1 score for entity-level and $36.31 \%$ F1 score for the surface forms.

Williams and Santia (2017) propose a statistical approach, where each word is associated with its context. Context conditional probabilities are used to estimate

\footnotetext{
${ }^{2}$ The details of the noisy dataset are given in Section 6.3.

${ }^{3}$ https://noisy-text.github.io/2017/
} 
the named entity tag probabilities. They obtain an F1 score of $26.30 \%$ on entitylevel and 25.26\% F1 score on surface forms. Jansson and Liu (2017), inspired by the work of Limsopatham and Collier (2016), use a bidirectional LSTM-CRF model that is similar to our baseline model but instead of orthographic features, Latent Dirichlet Allocation (LDA) (Blei et al., 2003) topic models and PoS tags are used as features. As a result, they achieve a performance of $39.98 \% \mathrm{~F} 1$ score on the entity-level and $37.77 \%$ F1 score on the surface forms.

\section{Neural Word Embeddings}

We use neural word embeddings of words as input to our proposed models. We use different levels of word embeddings such as the word-level word embeddings obtained by word2vec (Mikolov et al., 2013), character n-gram level word embeddings obtained by fasttext (Bojanowski et al., 2017), morpheme-level word embeddings obtained by morph2vec (Üstün et al., 2018), character-level embeddings, and orthographic character-level embeddings. By using these models, we aim to capture orthographic, morphological, and contextual information of words in noisy data.

For the notation that will be used throughout the article, we denote each sentence (i.e., tweet) by $S=\left(w_{1}, w_{2}, \ldots, w_{N}\right)$ that consists of $N$ tokens (i.e., words or other tokens), where the ith token is denoted by $w_{i}$.

\subsection{Orthographic Character-level Embeddings}

We use an orthographic character encoder similar to that of Aguilar et al. (2017) that encodes alphabetic characters as "c" (or "C" if the character is capitalized), numeric characters as " $\mathrm{n}$ ", punctuation as "p", and other characters as "x". For example, the word "Türkiye'ye!" (means to Turkey) becomes "Cccccccpccp". Each orthographic encoding is also padded with 0s accordingly with the longest word in the dataset to have a fixed length of orthographic embedding for all words. This allows us to reduce sparsity and capture the shapes and orthographic patterns within the words. We train the embeddings by a character-level CNN. We apply two-stacked convolutional layers and perform global average pooling on the output. Finally, we use a fully-connected feed-forward layer with a Rectifier Linear Unit (ReLU) activation function with the final character-level word representation of each word that is denoted by $E_{w_{i}}^{\left(c_{c n n}\right)}$. An overview of the architecture is given in Figure 1. Here, the word "Ankara!" is first encoded in terms of its characters such as "Cсссссp", and then the orthographic embeddings are fed into the convolutional layers to obtain the character representation for orthographic encoding.

As an alternative approach, we also train the orthographic character-level embeddings using a Bi-LSTM that is simply a combination of two different LSTMs (i.e., forward and backward LSTMs) where one of them takes the input sequence in the forward and the other one in the reverse order. Output of the forward and backward LSTMs are concatenated for the final orthographic character-level word embedding $E_{w_{i}}^{\left(c_{B i-L S T M}\right)}$. The Bi-LSTM model is given in Figure 2. Here, the sentence "29 ekimde Ankara'ya" is first encoded in terms of its orthographic characters such as 

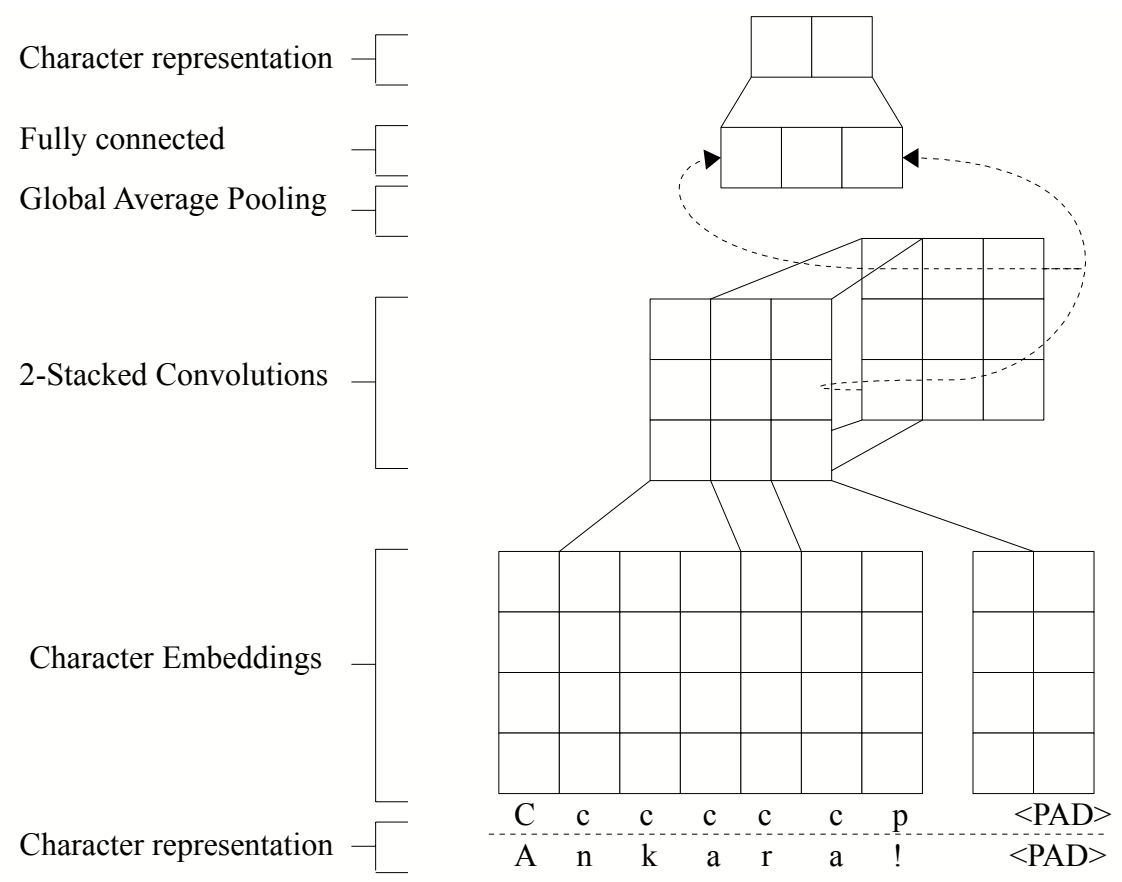

Fig. 1. Character-level word embedding using CNN. (Aguilar et al., 2017)

"nn cccccc Ccccccpcc", and then the embeddings of the orthographic characters are fed into a Bi-LSTM to obtain a character-level orthographic word embedding.

\subsection{Character-level Word Embeddings}

We also learn the character-level word embeddings using the actual characters rather than the character types unlike the orthographic word embeddings. For example, the word "Bravo" is first encoded in terms of the character embeddings of "B", "r", " $a "$, " $v "$, and "o".

We use another Bi-LSTM to learn the character-level word embeddings. To this end, the Bi-LSTM is fed by the character embeddings of the word. We obtain the character-level word embeddings denoted by $E_{w_{i}}^{(c)}$ by concatenating the vectors that are output by both LSTMs from both directions.

\subsection{Character N-gram-level Word Embeddings}

Fasttext (Bojanowski et al., 2017) is an extension of word2vec (Mikolov et al., 2013) and it is comparably better at capturing word representation for morphologicallyrich languages such as Turkish. This is due to its ability to form vector representation of words from their vectors of character n-grams. As a result, this allows us to generate word embeddings $E_{w_{i}}^{\left(c_{n g r a m}\right)}$ using n-grams even for out-of-vocabulary words which is a common case for noisy text and also agglutinative languages. 


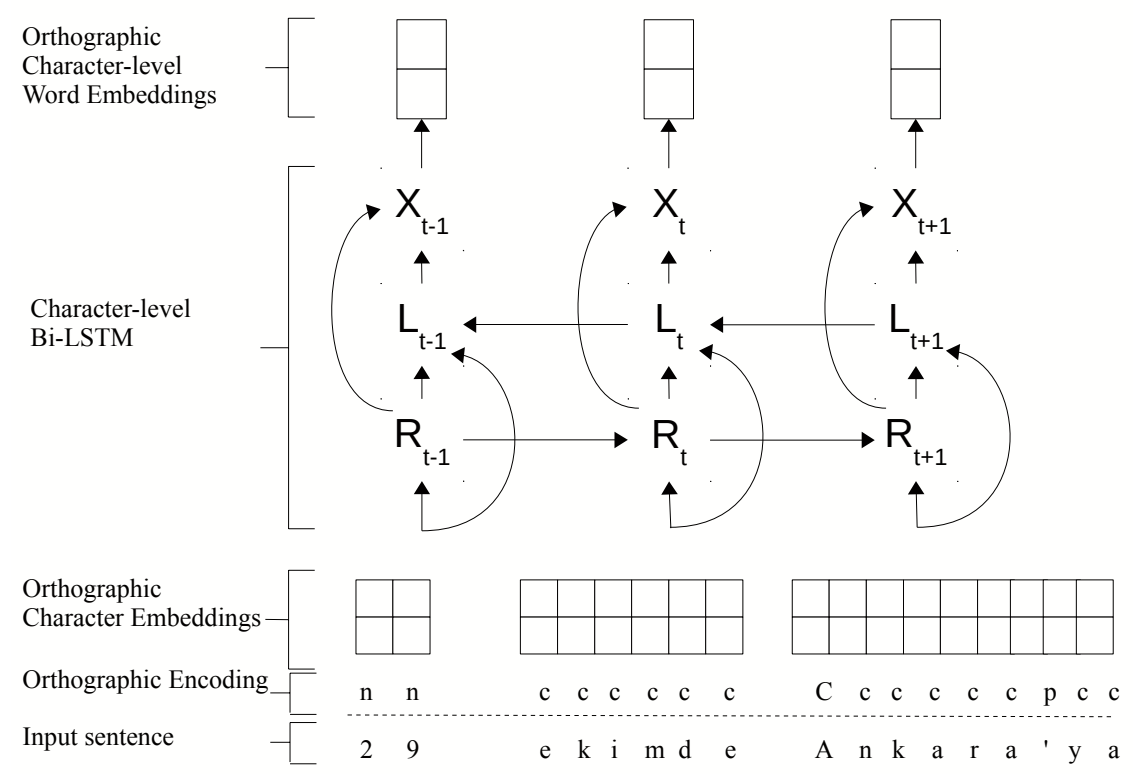

Fig. 2. Character-level word embedding using a bidirectional LSTM

\subsection{Morpheme-level Word Embeddings}

Morph2vec (Üstün et al., 2018) is another representation learning model that utilizes sub-word information to learn the word embeddings. The algorithm takes a list of candidate morphological segmentations of all words in the training data that are suggested by an unsupervised morphological segmentation system (Üstün and Can, 2016). Given that each word has multiple sequences of candidate morphological segmentations, the final word representation $E_{w_{i}}^{(m)}$ is a weighted sum of the morpheme-level word embeddings of all segmentations of that word. An attention mechanism is used on top of the model in order to learn the weights, where the mechanism assigns more weight to the correct segmentation of the word. We incorporate morpheme-level word embeddings that we obtain from pre-trained morph2vec embeddings in our proposed models in this article.

It can be argued that words in an informal text may not have proper morphemes. For example, "gidiyorum" in Turkish (means "I am going") is usually written as "gidiyom" by combining the present participle su $\mathrm{x}$-iyor with the person ending -um. However, morph2vec (Üstün et al., 2018) builds the word embeddings from several segmentations of the word that are likely to include the portions of the $\mathrm{su}$ xes in the corrupted form.

\subsection{Word-level Word Embeddings}

Word2vec (Mikolov et al., 2013) has been one of the leading word representation methods that has shown superior performance in capturing syntactic and semantic features of words. The method aims to estimate word embeddings $E_{w_{i}}^{(w)}$ using their 


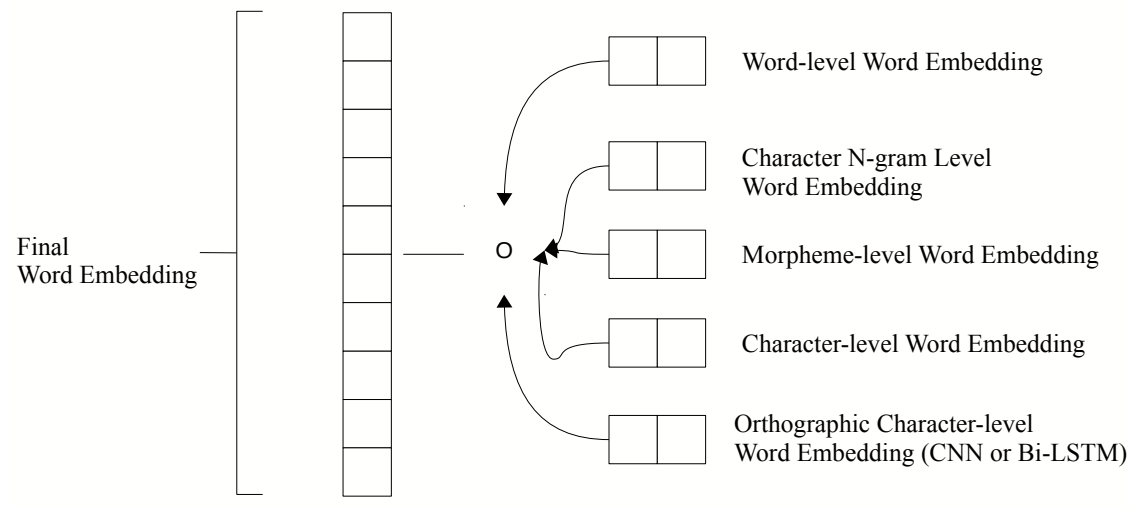

Fig. 3. Overview of the final word embeddings. After concatenating embeddings obtained from fasttext, word2vec, morph2vec, and character-level word embeddings, orthographic character-level embeddings, we apply dropout for better generalization.

contextual information similar to other aforementioned methods, but it does not make use of any subword information and all words are considered as distinct tokens.

\subsection{Final Word Embeddings}

The final word embeddings that we use as input to the proposed models are the concatenation of fasttext (Bojanowski et al., 2017), morph2vec (Üstün and Can, 2016), word2vec (Mikolov et al., 2013), character-level word embeddings, and orthographic character-level embeddings (either CNN-based or LSTM-based):

$$
E_{i}=E_{w_{i}}^{(w)} \quad E_{w_{i}}^{\left(c_{\text {ngram }}\right)} \quad E_{w_{i}}^{(m)} \quad E_{w_{i}}^{(c)} \quad E_{w_{i}}^{\left(c_{c n n \mid B i-L S T m}\right)}
$$

An overview of the approach is given in Figure 3. Each vertical stacked box represents a different level of word embedding for the given input word.

After concatenating the different-level word embeddings, we apply dropout on the final word embedding $E_{i}$. This prevents the model from solely depending on one type of word embedding and, therefore, ensures a better generalization. We assign dropout rate $r=0.5$.

\section{Baseline Model}

Our baseline model is founded on the well-known bidirectional LSTM-CRF (BiLSTM-CRF) model proposed for sequence labeling, which is similar to that of Huang et al. (2015); Chiu and Nichols (2015); Lample et al. (2016); Ma and Hovy (2016).

A Bi-LSTM is fed by the final word embeddings $E_{i}$ in order to learn the higherorder latent features for the named entity recognition task, and another layer with a 


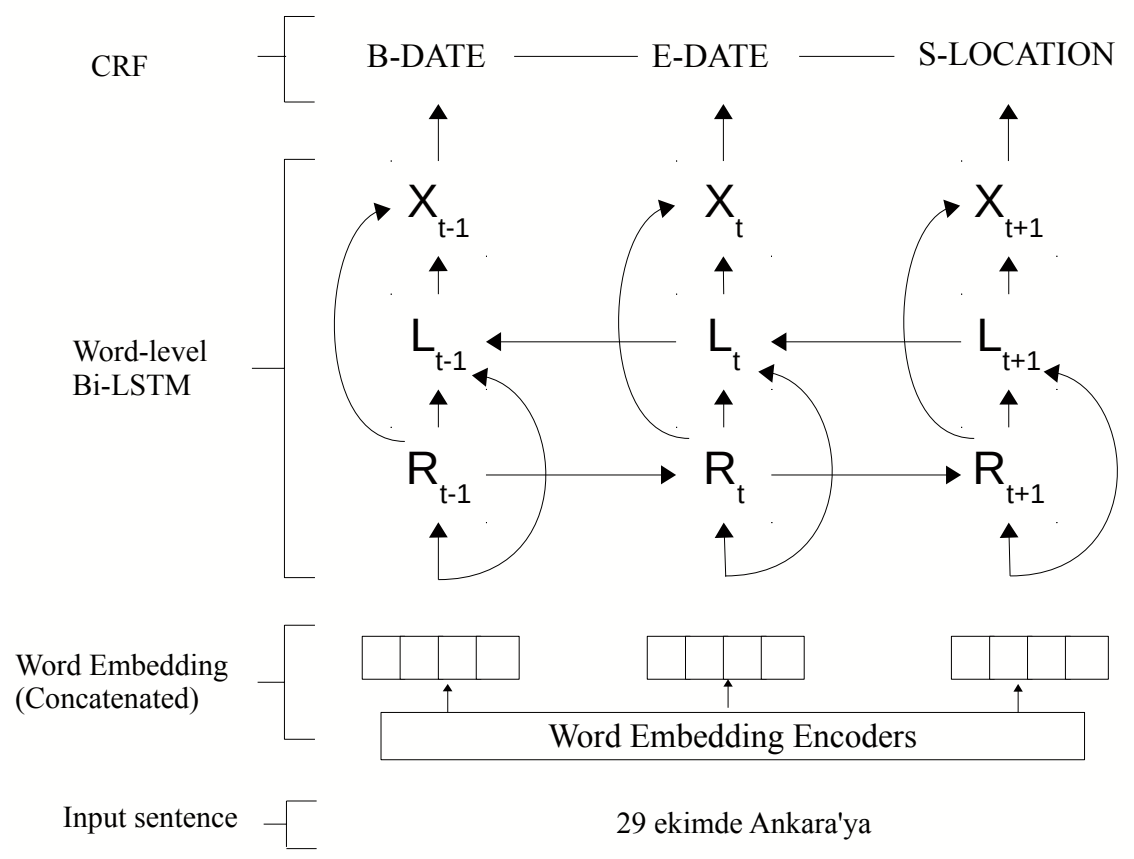

Fig. 4. Architecture of our baseline Bi-LSTM-CRF model. We learn latent features by using a Bi-LSTM that is fed by the combined word embeddings and then we feed the output of each Bi-LSTM state to CRF in order to predict the label sequence. Here, Word Embedding Encoders are namely word2vec (Mikolov et al., 2013),fasttext (Bojanowski et al., 2017), morph2vec (Üstün and Can, 2016), character-level word embedding, and orthographic character-level embedding methods. The Turkish input sequence "29 ekimde Ankara'ya" means "To Ankara on 29th October".

linear-chain CRF is fed by the LSTM outputs of each word to compute a prediction of the label sequence. Overview of the baseline model is given in Figure 4. Word embeddings are encoded as given in Figure 3.

\subsection{Bidirectional LSTM Layer}

Given an input sentence (i.e., tweet) $S=\left\{w_{1}, w_{2}, \ldots, w_{n}\right\}$ bidirectional LSTM is used to process the words sequentially. To this end, the combined word embed$\operatorname{ding} E_{i}$ of each word in the sentence is given as input to the bidirectional LSTM layer that is composed of a forward LSTM LSTM forward and a backward LSTM LST $M_{\text {backward }}$ (Hochreiter and Schmidhuber, 1997). Latent feature vectors $\bar{R}_{t} \rightarrow \overleftarrow{\text { and }}$ 
$L_{t}$ are learned as an output of the LSTMs at time step t:

$$
\begin{aligned}
& \bar{R}_{t} \rightarrow \leftarrow \operatorname{LSTM}_{\text {forward }}\left(E_{1: N}, t\right) \\
& L_{t}=\operatorname{LSTM} M_{\text {backward }}\left(E_{1: N}, t\right)
\end{aligned}
$$

The outputs of the LSTMs are concatenated to build a single vector output from the Bi-LSTM as follows:

$$
X_{t}=\bar{R}_{t} \rightarrow \overleftarrow{L}_{t}
$$

where $X_{t}$ denotes the concatenated output vector for each word. We also apply dropout on $X_{t}$ for a better generalization. Weights of the Bi-LSTM are initialized using uniform Glorot initialization (Glorot and Bengio, 2010) that initializes the weights by drawing samples from a Gaussian distribution with mean $=0.0$ and variance based on the fan-in (input units in the weight tensor) and fan-out (output units in the weight tensor) of the weight.

\subsection{CRF Layer}

We use a linear-chain Conditional Random Field (CRF) to predict the sequence of labels $Y=\left(y_{1}, y_{2}, \ldots, y_{N}\right)$ for the sentence $S$ where $y_{i}$ denotes the named entity label of the ith word $w_{i}$ in $S$. The prediction score of a sequence is defined as follows:

$$
C(S, Y)=\sum_{i=0}^{N} A_{y_{i}, y_{i+1}}+\sum_{i=1}^{N} P_{i, y_{i}}
$$

where the score is estimated over a sequence of size $N$. Here, $P$ is the matrix of scores that is output by the Bi-LSTM and $A$ is the matrix that denotes the transitions from the previous label to the next label. $P$ has a size of $N \cdot k$ where $k$ is the number of the distinct entity tags. The concatenated representation of each word $X_{t}$ is linearly projected onto a layer that has a size of $k$. Therefore, the matrix defines the scores of labelling each word in the sequence with the possible $k$ tags, which is not a proper probability distribution yet. In other words, $P_{i, y_{i}}$ is the score of the tag $y_{i}$ for a given a word $w_{i}$. By defining a log-linear model using the scores, the probability of the output sequence of $Y$ becomes:

$$
p(Y \mid S)=\frac{e^{C(S, Y)}}{\sum_{\widetilde{Y} \in Y_{S}} e^{C(S, Y)},}
$$

where $Y_{S}$ denotes the set of possible label sequences for $S$. Finally, the goal becomes to maximize the log-probability of the predicted label sequence. Building the loglinear model gives us the form:

$$
\log (p(Y \mid S))=C(S, Y) \quad \log \left(\sum_{\tilde{Y} \in Y_{S}} e^{C(S, \tilde{Y})}\right)
$$

The correctly-predicted sequence of labels is the one that maximizes Equation 7:

$$
\underset{\tilde{Y} \in Y_{s}}{\arg \max } C(S, \tilde{Y}) \text {. }
$$




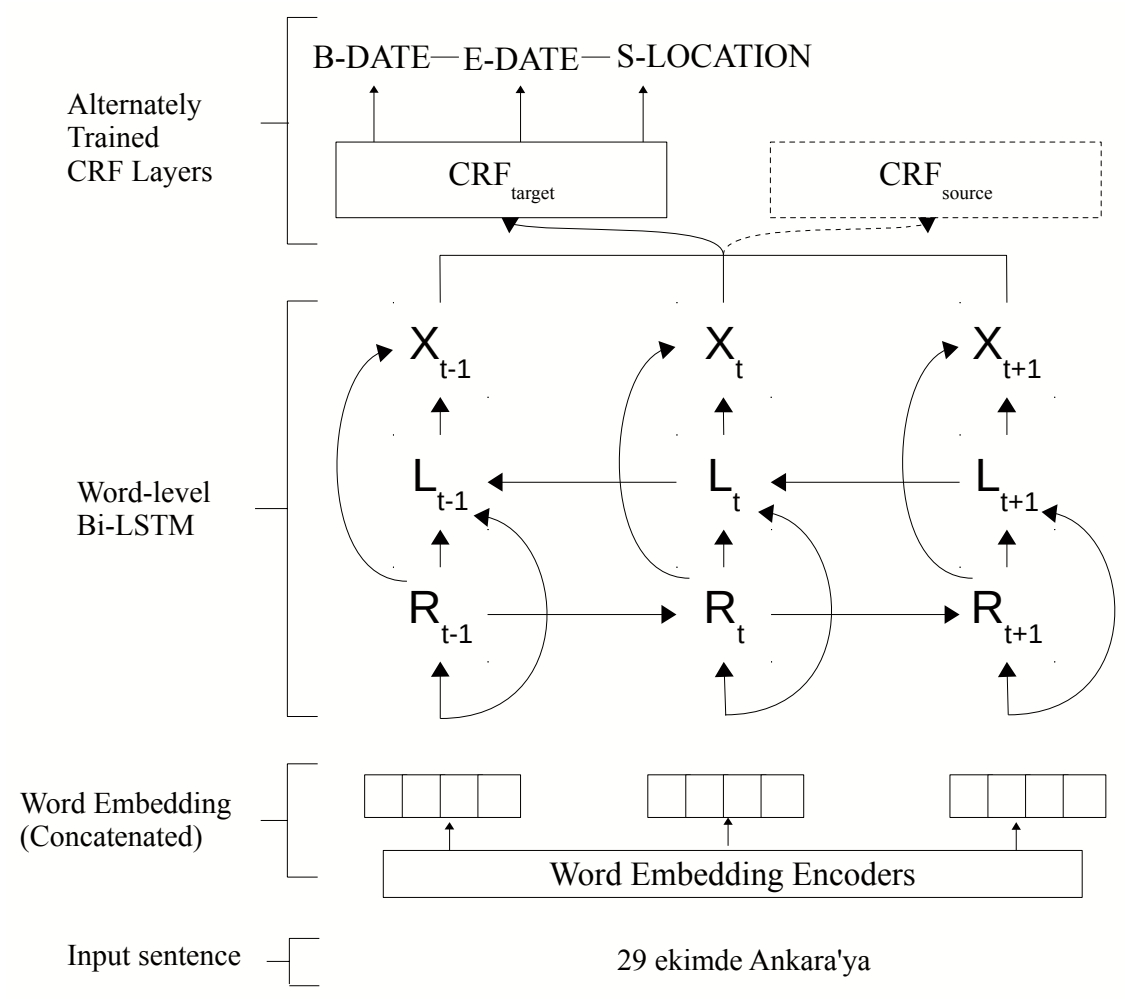

Fig. 5. Overview of the transfer learning model that incorporates an additional CRF layer. CRF layers are alternately trained on different datasets so that the shared layers learn from both datasets and therefore learning can be transferred from the source dataset to the target dataset. The Turkish input sequence "29 ekimde Ankara'ya" means "To Ankara on 29th October".

Weights of the CRF layers are initialized using uniform Glorot distribution. Both the parameter estimation and decoding are performed by dynamic programming.

\section{Transfer Learning}

The amount of annotated Turkish noisy text is considerably limited. This prevents the basic baseline model from learning especially some of the infrequent types such as DATE, TIME, and PERCENTAGE. To overcome this problem, we incorporate another CRF layer ( $\left.C R F_{\text {source }}\right)$ that is trained on a different, but preferably a larger dataset (i.e., source dataset), in addition to the $\mathrm{CRF}$ layer $\left(C R F_{\text {target }}\right)$ that is trained on a small amount of noisy text (i.e., target dataset). Therefore, the model learns from both datasets jointly.

The architecture of the baseline transfer learning model is given in Figure 5. As 


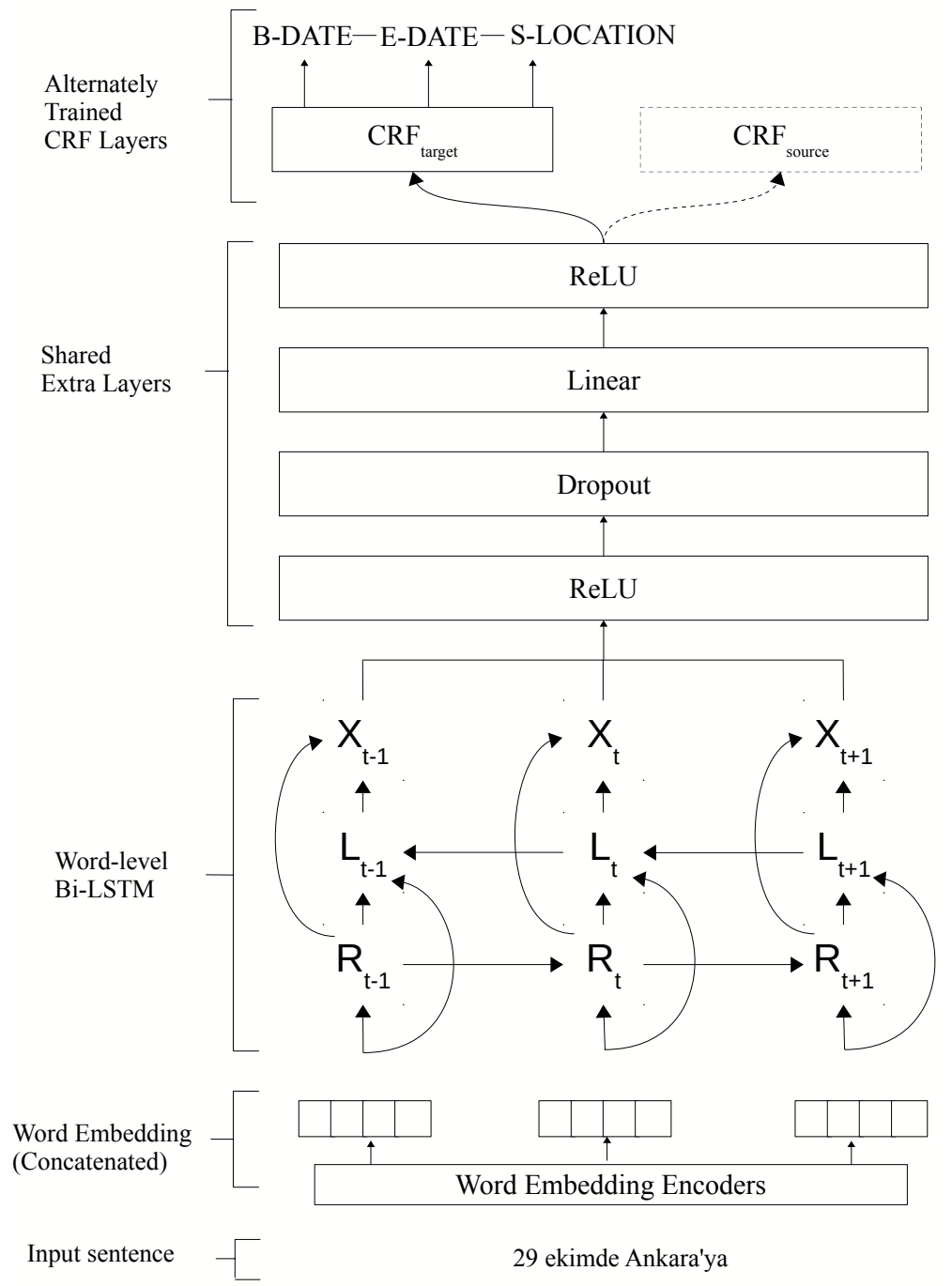

Fig. 6. Overview of the extended transfer learning model that incorporates an additional CRF layer. CRF layers are alternately trained on different datasets so that the shared layers learn from both datasets and therefore learning can be transferred from the source dataset to the target dataset. The Turkish input sequence "29 ekimde Ankara'ya" means "To Ankara on 29th October".

shown on the figure, lower layer that involves the word-level Bi-LSTM is shared by two CRFs. The embeddings are also shared by both CRF layers. However, the CRF layer involves two independent CRFs, where one of them is trained on the formal text and the other one is trained on the noisy text. Therefore, we transfer the de- 
pendencies learnt from the larger and formal text towards the noisy text gradually. The training procedure is performed by doing the gradient updates through each CRF layer alternately. In other words, in every other iteration the output of one CRF layer is considered to perform the gradient update based on its loss by discarding the output of the other CRF layer. Therefore, the outputs of both CRFs are used alternately, where both CRF outputs are gradually optimized in time. In this way, using the knowledge transferred from the larger text, some dependencies between rare entity types and rare words are also learnt for the noisy text.

This model is an adaptation of the cross-domain transfer learning model proposed by Yang et al. (2017). In their work, the authors introduce various transfer learning architectures for cross-domain, cross-application and cross-lingual transfer. We adapt the cross-domain transfer learning architecture by introducing the parameter sharing in the word-level Bi-LSTM, where each domain learns a separate CRF layer. However, the LSTMs are shared across different domains. Cotterell and Duh (2017) also apply a similar transfer learning scheme for low-resource named entity recognition with a shared Bi-LSTM across different languages with language-specific CRFs. We particularly used the Bi-LSTM-CRF architecture and not a single Bi-LSTM as suggested by Riedl and Padó (2018) for transfer learning because the best results have already been achieved by this architecture without transfer learning (Ma and Hovy, 2016; Cotterell and Duh, 2017; Reimers et al., 2014).

We further extended the transfer learning architecture by adding extra shared layers on the baseline architecture. Following the various architectures proposed by von Däniken and Cieliebak (2017), we added two Rectified Linear Units (ReLU), a dropout layer, and a linear layer (a feed-forward network) between the Bi-LSTM and the CRF layers. The architecture of the model is given in Figure 6. First, $X_{t}$, the output of the Bi-LSTM, is passed through a ReLU (Nair and Hinton, 2010) layer. Then, a dropout is applied to $X_{t}$. The dropout applied output $X_{t}$ is then passed through a feed-forward network with one hidden layer and ReLU activation, which outputs a score for possible $k$ number of entity tags:

$$
\text { score }_{t}=W_{2} \cdot \operatorname{Re} L U\left(W_{1} h_{t}+b_{1}\right)+b_{2}
$$

where $W_{1} \in R^{d_{H} \times d_{X}}, b_{1} \in R^{d_{H}}, W_{2} \in R^{k \times d_{H}}$, and $b_{2} \in R^{k}$ are the weights of the feed-forward network. Here, $d_{H}$ is the dimension of the hidden layer and $d_{X}$ is the dimension of $X_{t}$. As seen from the figure, all layers and their parameters are shared by both CRF layers. The motivation behind adding a feed-forward network between the Bi-LSTM layer and the CRF layer is to encode the outputs of the Bi-LSTM by introducing sparsity to lead the negative features to become zero. Otherwise, vanishing gradients problem stands out again due to the many layers that require backpropagation during gradient descent. Therefore, some outputs are forced to be zero by the ReLU unit and the vanishing gradient problem is naturally solved in this multi-layered architecture.

As for the training, analogously we performed backpropagation using the loss of one of the CRF layers alternately. Therefore, the CRF layer gains generalization through two different datasets from different domains during training. 


\section{Experiments \& Results}

We did the experiments for the baseline and the transfer learning models on Turkish, and additionally on English to compare with other related work. First, we describe the datasets, the implementation details of the models, and the evaluation methods that we followed in this work, then we present the experimental results along with a discussion on the results.

\subsection{Implementation Details}

Both the CNN-based $\left(E_{w_{i}}^{\left(c_{c n n}\right)}\right)$ and Bi-LSTM $\left(E_{w_{i}}^{\left(c_{B i} L S T m\right.}\right)$ based orthographic character embeddings have a dimensionality of 30 . The CNN-based character embeddings are initialized by uniform Glorot initializer. For the CNN model, 20 is assigned for the maximum word length, where the shorter words are padded with zeros and the longer ones are truncated. The Bi-LSTM based character-level word representation has a dimensionality of 60 .

We trained fasttext (Bojanowski et al., 2017) for Turkish with a learning rate of 0.025 for 4 epochs to learn the character n-gram-level word embeddings $E_{w_{i}}^{\left(c_{n g r a m}\right)}$ that have a dimensionality of 200. Character n-gram-level word embeddings have a dimension of 300 for English.

Morpheme-level word embeddings $E_{w_{i}}^{(m)}$ have a dimensionality of 75 and 50 for English and Turkish respectively. Word-level word embeddings $E_{w_{i}}^{(w)}$ have a dimension of 400 for both English and Turkish.

Weights of the shared ReLU and linear layers in transfer learning models are initialized using uniform Glorot initializer and biases are set to 0 .

During all experiments, both the baseline and transfer learning models are trained using backpropagation and the parameters are optimized using Stochastic Gradient Descent (SGD) algorithm. We trained both models for 100 epochs and set the learning rate to 0.005 in addition to using gradient clipping of 5.0. Dropout rate of all of the dropout layers are set to 0.5. Hidden dimension of character-level BiLSTM and word-level Bi-LSTM layers are set to 30 and 250 respectively. Tuning the dimensions or any other hyperparameter did not significantly improve the accuracy of the models. An overview of the hyperparameters is given in Table 1.

All models are implemented using Tensorflow 1.8.0 $0^{4}$ and the implementations and the related material are publicly available ${ }^{5}$.

\subsection{Tagging Scheme}

When it is thought that a named entity can span multiple consecutive words, a tagging scheme that impose some constraints on determining the possible label of a word is highly useful. IOB format is such a tagging scheme that uses $B$ for the

\footnotetext{
${ }^{4}$ https://www.tensorflow.org/

${ }^{5}$ All source code and related material are available on https://github.com/emrekgn/ turkish-ner.
} 
Table 1. Implementation and training details.

\begin{tabular}{|c|c|}
\hline Setting/Hyperparameter & Value \\
\hline gradient clip & 5.0 \\
\hline learning rate & 0.005 \\
\hline lr optimizer & sgd \\
\hline batch size & 10 \\
\hline dropout & 0.5 \\
\hline epochs & 100 \\
\hline hidden size Bi-LSTM(char) & 30 \\
\hline hidden size $_{\mathrm{Bi}-\mathrm{LSTM} \text { (word) }}$ & 250 \\
\hline dimension $_{\text {fasttext(en) }}$ & 300 \\
\hline dimension $_{\text {morph2vec(en) }}$ & 75 \\
\hline $\operatorname{dimension}_{\text {fasttext(tr) }}$ & 200 \\
\hline dimension $_{\text {morph2vec }(\operatorname{tr})}$ & 50 \\
\hline dimension $_{\text {word2vec }}$ & 400 \\
\hline dimension $_{\text {char }}$ & 30 \\
\hline
\end{tabular}

token that refers to the beginning of a named entity, $I$ for the token that refers to the inside of a named entity, and $O$ for the token for other words in the sequence. IOBES is a variant of IOB format that further restricts the possible label of a word with additional tokens such as $E$ token that is used for specifying the ending of a named entity, and $S$ token that is used for the named entities with only one word. Here is an example sentence tagged with the IOBES format:

Mustafa/B-PERSON Kemal/I-PERSON Atatürk/E-PERSON was born in $1881 / \boldsymbol{S}-\boldsymbol{D A T E}$ in the former Ottoman/B-ORGANIZATION Empire/EORGANIZATION.

We follow the IOBES tagging scheme for Turkish and IOB tagging scheme for English to be able to compare with other related work using the same annotated noisy text.

\subsection{Datasets}

In order to obtain Turkish character n-gram-level word embeddings, we trained Skipgram model of fasttext (Bojanowski et al., 2017) on a corpus of 20M Turkish tweets $^{6}$. As for English, we used the pre-trained English word embeddings that are provided by fasttext ${ }^{7}$ (Bojanowski et al., 2017). The word embeddings are obtained

\footnotetext{
${ }^{6}$ http://www.kemik.yildiz.edu.tr/data/File/20milyontweet.rar

7 https://s3-us-west-1.amazonaws.com/fasttext-vectors/crawl-300d-2M-subword.zip
} 
Table 2. Datasets

\begin{tabular}{ccccc}
\hline \hline$\#$ & Dataset & NE types & \# of tokens & \# of NEs \\
\hline DS-1 & TR-tweet & $\begin{array}{l}\text { ENAMEX, TIMEX, NUMEX } \\
\text { corporation, creative-work, } \\
\text { group, location, } \\
\text { DS-2 }\end{array}$ & WNUT'17 & person, product \\
\hline \hline
\end{tabular}

from the CBOW model of fasttext trained on Common $\mathrm{Crawl}^{8}$, a web site that provides web crawl data.

Pre-trained word embeddings obtained from word2vec (Mikolov et al., 2013) are used to learn the morpheme-level word embeddings by imitating them in morph2vec (Üstün et al., 2018).

We use pre-trained word2vec (Mikolov et al., 2013) embeddings that are trained on a corpus that involves 400M English tweets (Godin et al., 2015). As for Turkish, we use pre-trained word2vec embeddings that are trained on a news corpus (BOUN web corpus) that involves 423M words (Sak et al., 2008, 2011) and 20M Turkish tweets (Sezer et al., 2013).

We experimented on two datasets on Turkish and English that are given in Table 2. $D S-1$ (Şeker and Eryiğit, 2017) is the re-annotated version of the initial Turkish noisy dataset (Çelikkaya et al., 2013) that consists of ENAMEX, TIMEX, and NUMEX types. As we can see in Table 3 , this is a relatively small dataset with a highly imbalanced entity type distribution. Since the dataset does not have training and test splits, during experiments, we applied 10-fold cross validation and split the dataset into training, test and validation sets with a ratio of $80 \%, 10 \%, 10 \%$ respectively for Turkish and we did not apply a cross validation for English to be able to compare our results with other work participated in the 3rd Workshop on Noisy User-generated Text (WNUT'17) ${ }^{9}$.

$D S-2$ is an English noisy dataset (Derczynski et al., 2017) that is released by the 3rd Workshop on Noisy User-generated Text (WNUT'17) that includes person, location, corporation, product (consumer goods, service), creative work (song, movie, tv series, book), group (music band, sports team, non-corporate organizations) types. This dataset has training, test and development sets with a size of $65 \mathrm{~K}, 23 \mathrm{~K}$, and $16 \mathrm{~K}$ tokens. Distribution of the entity types in this dataset are also given in Table 4.

\subsection{Preprocessing}

Prior to tokenization of the datasets,

\footnotetext{
8 https://commoncrawl.org/

${ }^{9}$ https://noisy-text.github.io/2017/
} 
Transfer learning for Turkish named entity recognition on noisy text

Table 3. Number of entity types in Turkish noisy dataset, DS-1

\begin{tabular}{lr}
\hline \hline Entity Type & Amount \\
\hline person & 699 \\
location & 230 \\
organization & 363 \\
date & 56 \\
time & 20 \\
money & 12 \\
percentage & 3 \\
\hline Total & 1,383 \\
\hline
\end{tabular}

Table 4. Number of entity types in English noisy dataset, DS-2

\begin{tabular}{lrrrr}
\hline \hline Entity Type & Train & Development & Test & Total \\
\hline person & 660 & 470 & 429 & 1,559 \\
location & 548 & 74 & 150 & 772 \\
corporation & 221 & 34 & 66 & 321 \\
product & 142 & 114 & 127 & 383 \\
creative-work & 140 & 104 & 142 & 386 \\
group & 264 & 39 & 165 & 468 \\
\hline Total & 1,975 & 835 & 1,079 & 3,889 \\
\hline \hline
\end{tabular}

- We replaced the URLs (tokens starting with http) with a special token. This allows us to reduce sparsity and allows our model to converge relatively faster.

- We replaced the Twitter mentions (Twitter usernames starting with@ sign) with another special token in DS-1. This reduced the number of PERSON entities from 4256 to 699 and we believe this prevents memorizing the mentions in the text.

\subsection{Evaluation Methods}

We evaluate the results with accuracy, precision and recall. Accuracy measures the overall performance of the model by computing the ratio of correctly labeled tokens to the total number of tokens. However, this results in a highly imbalanced value since most of the tokens are not part of a named entity and, therefore, labeled as OTHER. Precision gives the ratio of correctly labeled named entities (chunks) to the total label predictions and recall measures the ratio of correctly labeled named 
Table 5. Overview of the experimental results of the baseline models on the Turkish noisy dataset, DS-1. Baseline-2 uses extra layers in the Bi-LSTM CRF model. Fasttext (Bojanowski et al., 2017), morph2vec (Üstün et al., 2018), word2vec Mikolov et al. (2013), character-level and orthographic embeddings are denoted in the embeddings column by ft, m2v, w2v, char and ortho respectively. Acc refers to accuracy, $P$ refers to Precision, and $R$ refers to Recall.

\begin{tabular}{llllll}
\hline \hline Model & Embeddings & Acc. (\%) & P (\%) & R (\%) & F1 (\%) \\
\hline baseline & char & 96.81 & 48.16 & 18.05 & 26.12 \\
baseline & m2v & 96.66 & 63.68 & 11.34 & 19.14 \\
baseline & m2v, char & 96.70 & 47.78 & 14.29 & 21.88 \\
baseline & m2v, ortho & 96.72 & 60.6 & 14.11 & 22.81 \\
baseline & $\mathrm{ft}$ & 97.69 & 72.42 & 49.79 & 58.91 \\
baseline & $\mathrm{ft}$, char & 97.7 & 74.25 & 48.69 & 58.69 \\
baseline & $\mathrm{ft}$, ortho & 97.73 & 71.96 & 51.10 & 59.7 \\
baseline & $\mathrm{ft}, \mathrm{m} 2 \mathrm{v}$ & 97.67 & 71.36 & 50.02 & 58.73 \\
baseline & $\mathrm{ft}, \mathrm{m} 2 \mathrm{v}$, char & 97.72 & 74.48 & 49.11 & 59.03 \\
baseline & $\mathrm{ft}, \mathrm{m} 2 \mathrm{v}$, ortho & 97.68 & 73.12 & 48.70 & 58.32 \\
baseline & $\mathrm{ft}, \mathrm{m} 2 \mathrm{v}$, ortho (cnn) & 97.69 & 74.00 & 49.46 & 59.07 \\
baseline & w2v & 96.73 & 68.49 & 13.23 & 22.02 \\
baseline & w2v, char & 96.77 & 53.28 & 19.57 & 28.45 \\
baseline & w2v, ortho & 96.69 & 60.64 & 16.57 & 25.8 \\
baseline & w2v, m2v & 97.08 & 65.34 & 27.63 & 38.68 \\
baseline & w2v, m2v, char & 97.13 & 63.08 & 30.31 & 40.78 \\
baseline & w2v, ft & 97.77 & 71.29 & 53.26 & 60.82 \\
baseline & w2v, ft, char & 97.75 & 70.91 & 53.19 & 60.58 \\
baseline & w2v, ft, m2v & 97.68 & 73.49 & 47.45 & 57.53 \\
baseline & w2v, ft, m2v, char & 97.80 & 70.71 & 52.31 & 59.98 \\
baseline & w2v, ft, m2v, ortho & $\mathbf{9 7 . 8 1}$ & $\mathbf{7 3 . 6 5}$ & $\mathbf{5 2 . 9 9}$ & $\mathbf{6 1 . 5 3}$ \\
baseline-2 & w2v, ft, m2v, ortho & 97.51 & 69.00 & 53.00 & 60.15 \\
& & & & & \\
\hline \hline
\end{tabular}

entities (chunks) to the total number of correct predictions. Finally, F1 score is computed as the harmonic mean of precision and recall:

$$
F 1=\frac{2 * \text { precision } * \text { recall }}{(\text { precision }+ \text { recall })}
$$

In order to measure the overall performance of any given model for the sequence labelling task, F1 score is commonly chosen over accuracy since it intuitively defines a good measure of the model by taking false negatives and false positives into account, whereas accuracy gives imbalanced results due to highly-skewed entity type distribution because most of the tokens do not have an entity label.

\subsection{Experimental Results on Turkish}

We experimented with different combinations of embedding methods to analyze the impact of the word and subword embedding methods used in the baseline and 
the transfer learning models. To this end, we used word-based word embedding method word2vec Mikolov et al. (2013), character n-gram level word embedding method fasttext (Bojanowski et al., 2017), morpheme-level word embedding method morph2vec (Üstün and Can, 2016), character embeddings trained with a Bi-LSTM (and $\mathrm{CNN}$ ), and orthographic character-level embeddings trained on a characterlevel Bi-LSTM.

The results obtained from the baseline model are given in Table 5 . In the baseline model, amongst using only one type of embedding, fasttext performs the best compared to other embedding types with a F1 measure of $58.91 \%$, where CNN-based orthographic char embeddings performs $26.12 \%$, morph2vec performs $19.14 \%$, and word2vec performs $22.02 \%$. This shows that using character n-grams in representation learning can cope with the sparsity issue better compared to other embedding types. We were expecting a similar performance from the morph2vec embeddings, however they have not performed as well as fasttext. This might be a sign of ill-formed nature of the noisy text, where the morphemes are degenerated. Since morph2vec is trained on a formal text with exact morpheme boundaries, the noisy text could not benefit from the morphological knowledge adequately.

When the contribution of other embeddings used along with fasttext embeddings is observed, we see that orthographic features contribute the most with an improvement of $0.79 \%$ and the other embedding types do not contribute to the performance of the model and rather they degrade the results. We believe that since fasttext embeddings contain also character-level and morpheme-level features, those embeddings do not provide a significant improvement on the fasttext embeddings. The results are also similar when more embeddings are combined with fasttext embeddings, which is due to a similar reason.

Because of the morphological structure of Turkish, using solely word2vec trained word embeddings do not perform very well. Combining the word embeddings with character embeddings or orthographic embeddings improves the scores, although the final scores are still below $30 \%$. Using morph2vec along with word2vec provides a better improvement compared to character-level word embeddings and orthographic embeddings with a F1 measure of $38.68 \%$. The highest improvement is obtained when word2vec is combined with fasttext and it gives a F1 measure of $60.82 \%$.

The highest performance is obtained when all embedding types (fasttext, word2vec, morph2vec and orthographic encoding) are used together, which gives an F1 measure of $61.53 \%$. The highest scores obtained for different entity types are given in Table 6. Our baseline model fails to label the infrequent types such as time, money, and percentage since the annotated noisy data is too small to learn the latent features for such infrequent entity types. However, the frequent entity types such as person and organization are learned well compared to location and date.

In order to transfer any learned features from another larger dataset, we added an extra CRF layer where the Bi-LSTM layers are shared by both datasets as described in Section 5. We call this model transfer learning 1. We trained the model alternately with different datasets in each epoch. Therefore the shared layers up to the CRF layers can learn from both of the datasets. As a larger dataset 
Table 6. Experimental results of the baseline model with fasttext (Bojanowski et al., 2017), morph2vec (Üstün et al., 2018), word2vec (Mikolov et al., 2013) and orthographic character-level embeddings on Turkish noisy dataset, DS-1

\begin{tabular}{lrrr}
\hline \hline Entity Type & Precision (\%) & Recall (\%) & F1 score (\%) \\
\hline person & 70.61 & 54.10 & 61.17 \\
organization & 83.28 & 63.81 & 71.85 \\
location & 68.36 & 46.28 & 54.84 \\
date & 45.71 & 27.57 & 32.85 \\
time & 0 & 0 & 0 \\
money & 0 & 0 & 0 \\
percentage & 0 & 0 & 0 \\
\hline overall & $\mathbf{7 3 . 6 5}$ & $\mathbf{5 2 . 9 9}$ & $\mathbf{6 1 . 5 3}$ \\
\hline \hline
\end{tabular}

(source dataset), we used the re-annotated version of the Turkish news corpus with $492 \mathrm{~K}$ tokens which was originally provided by Tür et al. (2003) and re-annotated by Şeker and Eryigit (2017). The results obtained from the transfer learning models are given in Table 7. By using orthographic character-level word embeddings, character n-gram-level word embeddings, morpheme-level word embeddings and word-level word embeddings we obtained an F1 score of $66.17 \%$ that is better than the baseline model that incorporates all embedding types.

We also incorporated additional ReLU and linear layers between the Bi-LSTM and CRF layers as described in Section 5. We call the extended model as transfer learning 2. The results obtained from the transfer learning model are coherent with the results of the baseline model. Fasttext embeddings perform the best with a F1 measure of $62.47 \%$, whereas using the other embedding types on its own perform comparably poorer similar to the baseline model. Morph2vec embeddings and character embeddings perform alike with a $\mathrm{F} 1$ measure of $34.62 \%$ and $36.74 \%$ respectively, which are still significantly better than the results obtained from the baseline model when those embeddings are used alone. This is possibly due to the inclusion of another larger dataset that compensates the sparsity issue in embeddings.

Interestingly, using character embeddings in addition to fasttext embeddings improves the F1 score from $62.47 \%$ to $64.09 \%$, whereas in the baseline model adding character embeddings on fasttext embeddings did not make an impact. This is possibly due to the transfer of character embeddings between different domains. However, without transferring any character information between the domains, the fasttext emnbeddings seem to cover character embeddings and this hinders the impact of character embeddings against fasttext embeddings. Similar to the baseline results, using word2vec embeddings or character embeddings along with fasttext 
Table 7. Overview of the experimental results of the transfer learning models on the Turkish noisy dataset, DS-1. Fasttext (Bojanowski et al., 2017), morph2vec (Üstün et al., 2018), word2vec Mikolov et al. (2013), character-level and orthographic embeddings are denoted in the embeddings column by ft, m2v, w2v, char and ortho respectively. Transfer learning - 1 represents the basic transfer learning architecture without the additional (ReLU, linear) layers between the word-level Bi-LSTM and CRF layers and transfer learning - 2 is the transfer learning model with additional ReLU and linear layers. Acc refers to accuracy, $P$ refers to Precision, and $R$ refers to Recall.

\begin{tabular}{llllll}
\hline \hline Model & Embeddings & Acc. $(\%)$ & $\mathrm{P}(\%)$ & $\mathrm{R}(\%)$ & $\mathrm{F} 1(\%)$ \\
\hline transfer learning-1 & w2v, ft, m2v, ortho & 98.03 & 71.00 & 62.00 & 66.17 \\
transfer learning-2 & char & 96.91 & 50.98 & 28.87 & 36.74 \\
transfer learning-2 & $\mathrm{m} 2 \mathrm{v}$ & 96.9 & 53.62 & 25.84 & 34.62 \\
transfer learning-2 & $\mathrm{m} 2 \mathrm{v}$, char & 97.04 & 65.96 & 24.76 & 35.92 \\
transfer learning-2 & $\mathrm{ft}$ & 97.77 & 69.8 & 56.94 & 62.47 \\
transfer learning-2 & $\mathrm{ft}, \mathrm{char}$ & 97.82 & 69.29 & 59.73 & 64.09 \\
transfer learning-2 & $\mathrm{ft}, \mathrm{m} 2 \mathrm{v}$ & 97.81 & 67.78 & 61.48 & 64.27 \\
transfer learning-2 & $\mathrm{ft}$, ortho & 97.88 & 68.09 & 63.04 & 65.37 \\
transfer learning-2 & $\mathrm{ft}$, ortho (cnn) & 97.89 & 69.89 & 60.56 & 64.73 \\
transfer learning-2 & $\mathrm{ft}, \mathrm{m} 2 \mathrm{v}$, ortho & 97.87 & 70.78 & 60.35 & 65.12 \\
transfer learning-2 & $\mathrm{ft}, \mathrm{m} 2 \mathrm{v}$, ortho (cnn) & 97.95 & 74.45 & 58.94 & 65.72 \\
transfer learning-2 & w2v & 96.80 & 65.82 & 16.20 & 25.86 \\
transfer learning-2 & w2v, char & 96.95 & 59.97 & 23.77 & 33.75 \\
transfer learning-2 & w2v, m2v & 97.38 & 65.38 & 40.15 & 49.60 \\
transfer learning-2 & w2v, m2v, char & 97.45 & 66.28 & 40.73 & 50.34 \\
transfer learning-2 & w2v, ft & 97.89 & 70.09 & 59.86 & 64.46 \\
transfer learning-2 & w2v, ft, char & 97.91 & 69.47 & 61.66 & 65.18 \\
transfer learning-2 & w2v, ft, m2v & 97.88 & 68.19 & 61.53 & 64.64 \\
transfer learning-2 & w2v, ft, m2v, char & 97.86 & 68.58 & 60.38 & 64.19 \\
transfer learning-2 & w2v, ft, m2v, ortho & $\mathbf{9 8 . 0 0}$ & $\mathbf{7 1 . 7 9}$ & $\mathbf{6 3 . 9}$ & $\mathbf{6 7 . 3 9}$ \\
\hline \hline
\end{tabular}

embeddings improves the scores by around 2\%. Using orthographic embeddings along with fasttext embeddings also improves the scores by around $3 \%$.

Using character embeddings in addition to fasttext and word2vec embeddings still improves the scores with a F1 measure of $65.18 \%$, which was not the case in the baseline model. The highest score is obtained with a F1 measure of $67.39 \%$ when again the combination of all embedding types (word2vec, fasttext, morph2vec, orthographic embeddings) is used. Therefore, adding extra layers improved the results considerably.

As an alternative to orthographic character-level embeddings, we also incorporated the character-level embeddings that are trained on a character-level Bi-LSTM (by using the actual characters this time instead of replacing the characters with various symbols for the shape of the word) following the work of Lample et al. 
Table 8. Experimental results of transfer learning model (transfer learning - 2) with fasttext (Bojanowski et al., 2017), morph2vec (Üstün et al., 2018), word2vec (Mikolov et al., 2013) and ortographic character-level embeddings on Turkish noisy dataset, $D S-1$

\begin{tabular}{lrrr}
\hline \hline Entity Type & Precision (\%) & Recall (\%) & F1 score (\%) \\
\hline person & 71.20 & 65.52 & 67.95 \\
organization & 82.05 & 70.23 & 75.16 \\
location & 67.05 & 64.29 & 65.27 \\
date & 40.47 & 35.69 & 35.86 \\
time & 10.00 & 4.00 & 5.71 \\
money & 5.00 & 6.66 & 5.71 \\
percentage & 0 & 0 & 0 \\
\hline \multirow{2}{*}{ overall } & $\mathbf{7 1 . 7 9}$ & $\mathbf{6 3 . 9}$ & $\mathbf{6 7 . 3 9}$ \\
\hline \hline
\end{tabular}

(2016). However, the results obtained from the character-level word embeddings performed comparably poorer.

Additionally, in order to analyze the impact of the additional layers, we performed a separate experiment for the baseline model with a single CRF layer without any transfer learning. The model is called baseline-2 in Table 5 . We used word2vec, fasttext, morph2vec and orthographic embeddings in this setting. The baseline model with the additional layers gives $60.15 \% \mathrm{~F} 1$ score, which is lower than the baseline model without the additional layers using the same embeddings.

Table 8 presents the highest scores obtained for different entity types in the transfer learning model. We can see that the transfer learning model improves upon the results of the baseline model significantly. Although, the overall results on rare entity types (such as date, time, money) are higher compared to the baseline model, transfer learning model still fails to label percentage but we believe that this is an expected outcome given that it has only 3 examples belonging to the percentage type in the whole dataset. Note that we are also using cross-validation so that number of times an entity type is seen in one iteration is further decreased.

We trained Skipgram model of fasttext (Bojanowski et al., 2017) on the same corpus of 20M Turkish tweets ${ }^{10}$ for different dimensions of character n-gram level word embeddings to analyze the impact of the character n-gram level word embeddings' dimensionality. The results for different sizes of embeddings are given in Table 9. The results show that the scores improve with higher dimensionalities, where we obtain the highest scores with 200 dimensional fasttext embeddings. The results also support the findings of Yin and Shen (2018), where it was reported that the over-parametrization does not hurt the performance and the performance increases

10 http://www.kemik.yildiz.edu.tr/data/File/20milyontweet.rar 
Table 9. Experimental results obtained from different dimensions of fasttext character n-gram level word embeddings (Bojanowski et al., 2017)

\begin{tabular}{lrrrr}
\hline \hline Dimensionality & Accuracy & Precision (\%) & Recall (\%) & F1 score (\%) \\
\hline 50 dimensions & 97.17 & 74.26 & 27.56 & 40.05 \\
100 dimensions & 97.52 & 74.42 & 39.86 & 51.86 \\
150 dimensions & 97.60 & 73.36 & 44.94 & 55.53 \\
200 dimensions & $\mathbf{9 7 . 6 9}$ & $\mathbf{7 2 . 4 2}$ & $\mathbf{4 9 . 7 9}$ & $\mathbf{5 8 . 9 1}$ \\
250 dimensions & 97.68 & 72.93 & 48.50 & 58.15 \\
\hline \hline
\end{tabular}

with the dimensionality up to a level, where it degrades slightly and converges so long as the dimensionality increases.

In all experiments we used orthographic and character embeddings that have a dimensionality of 30 . We did further experiments to analyze the impact of the dimensionality of the orthographic embeddings. We used 200 dimensional fasttext embeddings along with orthographic embeddings with different dimensions $(30,50$, 100). However, the results did not change considerably and the F1 score was always around $59-60 \%$, which are in line with the previous results reported in Table 5.

Since we used pre-trained word2vec embeddings that are already highdimensional (400) we did not perform further experiments to analyze the dimensionality of the word2vec embeddings. As Yin and Shen (2018) suggests, the higher dimensions of word embeddings perform better compared to lower dimensions to a certain extent.

As for the morph2vec Üstün et al. (2018) embeddings, they are optimized by the authors using 50 and 75 for the morph vector dimensions for Turkish and English respectively.

\subsubsection{Comparison with Related Work on Turkish}

We compare our results with the related work on Turkish noisy dataset $D S-1$. The results are given in Table 10. The related work uses different re-annotated versions of the same dataset, therefore named entity distributions within the datasets may slightly differ, however the difference between the datasets is not very significant. Therefore, all results are comparable with each other. Şeker and Eryiğit (2017) present the latest version called $D S-1 v 4$, which is also used in our experiments. Note that, we replaced any Twitter mentions (number of mentions labelled as person: 3557 ) in the dataset prior to training. We compare our model with the results of Şeker and Eryiğit (2017), that are obtained by replacing the mentions in the tweets, so that we can make a fair comparison. Additionally, we compare our model with the models proposed by Çelikkaya et al. (2013), Küçük and Steinberger (2014), and Eken and Tantuğ (2015).

It should be noted that none of the related work on Turkish named entity recog- 
Table 10. Comparison with related work on Turkish noisy dataset DS-1. All results are tested on the same noisy text and are therefore comparable with each other.

\begin{tabular}{lrc}
\hline \hline Related Work & F1 score (\%) & Dataset \\
\hline Şeker and Eryiğit (2017) & 63.63 & DS-1 v4 \\
Çelikkaya et al. (2013) & 19.28 & DS-1 v1 \\
Küçük and Steinberger (2014) & 46.93 & DS-1 v2 \\
Eken and Tantuğ (2015) & 28.53 & DS-1 v3 \\
& & \\
\hline baseline (ft, m2v, w2v, ortho) & 61.53 & DS-1 v4 \\
baseline-2 (ft,m2v,w2v, ortho) & 60.15 & DS-1 v4 \\
transfer learning-1 (ft,m2v,w2v, ortho) & 66.17 & DS-1 v4 \\
transfer learning-2 (ft, m2v, w2v, ortho) & $\mathbf{6 7 . 3 9}$ & DS-1 v4 \\
\hline \hline
\end{tabular}

nition is designed particularly for noisy text. Therefore, those models are trained on formal text and, as an additional experimental setting, the authors also present their results on noisy text by using the Turkish noisy text (from $D S-1 v 1$ to $D S-1$ v4) only for testing purposes. Therefore, our training sets are different.

Our baseline model and the transfer learning model without the additional layers outperform the models proposed by Çelikkaya et al. (2013), Küçük and Steinberger (2014), and Eken and Tantuğ (2015) significantly with a F1 measure of $61.53 \%$ and $66.17 \%$ respectively, whereas the highest score among the other works is $46.93 \%$. The model proposed by Şeker and Eryiğit (2017) is slightly better with a F1 measure of $63.63 \%^{11}$. Nevertheless, our transfer learning model with extra layers outperforms all of the models with a F1 score of $67.39 \%$.

\subsubsection{Error Analysis}

We did a qualitative error analysis to examine the common errors in the results. Frequent person names are usually tagged correctly. However, if they are not frequent or if they are spelled with multiple vowels to give a shouting effect (e.g. Tülaaaaaaaayy, where the correct name is Tülay), then they may not be tagged correctly. In some circumstances the location names are also tagged as PERSON especially when the person names are followed straight away by location names. This mistagging does not occur when organization names are followed by location names.

Another frequent error type occurs when the organization names span across few

${ }^{11}$ Although $67.96 \%$ is reported by Şeker and Eryiğit (2017), this score is obtained by including Twitter mentions in both training and test data. Twitter mentions appear in almost any tweet which are easy to detect and therefore increase the scores naturally. Therefore, we compare our results with their score without using Twitter mentions to have a fair comparison. 
Table 11. A list of incorrect tags in Turkish

\begin{tabular}{|c|c|}
\hline & Examples \\
\hline $\begin{array}{l}\text { Predicted } \\
\text { Correct }\end{array}$ & $\begin{array}{l}\text { Ege } \backslash \text { S-LOCATION Üniversitesi } \backslash \text { O Bölümü } \backslash O \\
\text { Ege } \backslash \text { B-LOCATION } \quad \text { Üniversitesi } \backslash \text { I-LOCATION } \\
\text { LOCATION }\end{array}$ \\
\hline Predicted & 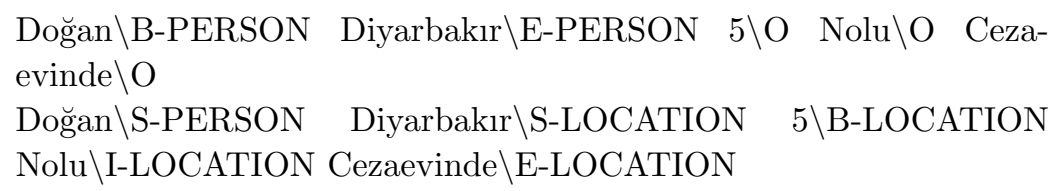 \\
\hline $\begin{array}{l}\text { Predicted } \\
\text { Correct }\end{array}$ & $\begin{array}{l}\text { Ziraat } \backslash \text { S-LOCATION Türkiye } \backslash \text { S-LOCATION Kupası } \backslash O \\
\text { Ziraat } \backslash \text { B-ORGANIZATION Türkiye } \backslash \text { I-ORGANIZATION } \quad \text { Kupası } \\
\backslash \text { E-ORGANIZATION }\end{array}$ \\
\hline $\begin{array}{l}\text { Predicted } \\
\text { Correct }\end{array}$ & 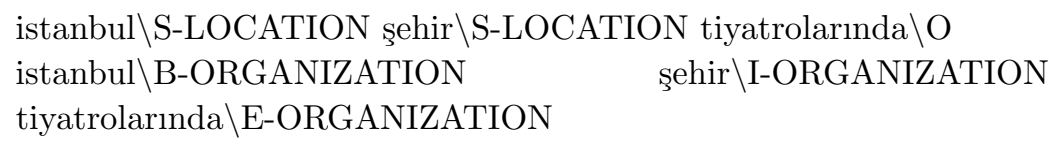 \\
\hline $\begin{array}{l}\text { Predicted } \\
\text { Correct }\end{array}$ & $\begin{array}{l}\text { FENERBAHCEEE } \backslash \mathrm{O} \\
\text { FENERBAHCEEE } \backslash \text { S-ORGANIZATION }\end{array}$ \\
\hline $\begin{array}{l}\text { Predicted } \\
\text { Correct }\end{array}$ & $\begin{array}{l}\text { bu } \backslash \text { O hafta } \backslash \text { O cuma } \backslash \text { S-DATE } \\
\text { bu } \backslash \text { B-DATE hafta } \backslash \text { I-DATE cuma } \backslash \text { E-DATE }\end{array}$ \\
\hline $\begin{array}{l}\text { Predicted } \\
\text { Correct }\end{array}$ & $\begin{array}{l}\text { Gizemcim } \backslash \mathrm{O} \\
\text { Gizemcim } \backslash \text { S-PERSON }\end{array}$ \\
\hline
\end{tabular}

words. Those organization names are usually confused with the location names. This mistagging also occurs when the organization name is not frequent enough. Another interesting usage is seen with the organization names that are shortened by the name of the location since the organization belongs to that location. For example, instead of using Trabzonspor (the football team that belongs to the city Trabzon), it is shortened to Trabzon to refer to the team. This requires more information to extract the correct meaning of the named entity and usually such names are mistagged by our models. Those are the typical tagging errors of the organization entries. Apart from these, frequent and single word organization names are tagged correctly by the models. Abbreviated organization names are also tagged correctly whether or not capitalized (e.g. 'FB' for the football team name 'Fenerbahçe', 'gs' for the football team name 'Galatasaray'). Even some organization names that include spelling errors are tagged correctly by our model. However, some of the 
misspelled organization names are not tagged as organization, but instead tagged as other in the gold data. Therefore, although those organization names are tagged correctly by our model, they are counted wrong. For example, Fenev (the name of the football team Fener is misspelt) is tagged as organization correctly by our model.

Location names that span across few words also usually cannot be identified properly and only the first word is tagged correctly. Infrequent location names are also tagged incorrectly. Another error occurs because of the non-Ascii characters in the location names. Since we do not perform any preprocessing on the data, those location names also cannot be identified correctly.

The inflection of named entities also have a significant impact on tagging. The inflectional morphemes such as case markers or possessive morphemes are seen frequently with the location names. To our observation, the frequent inflectional morphemes do not affect the tagging. For example, "samsunsporuma" (means "to my team samsunspor") is tagged correctly even though it has got two inflectional su xes (i.e., ' $u m$ ' for ' $m y$ ' and ' $a$ ' for 'to'). However, infrequent morphemes lead to mistagging with the location names. Person names are also sometimes inflected with the su x ciğim (means 'dear' and usually abbreviated as cim in the informal text) as a salutation and they cannot be tagged correctly.

The infrequent named entities are learned better in transfer learning, which is an expected result. Even some of the frequent named entities that are inflected can be correctly tagged in transfer learning model. Otherwise, the errors are common in baseline and transfer learning. Therefore, the main contribution of transfer learning is the compensation of the infrequent named entities using a larger corpus. When we also compare the results obtained from different levels of word embeddings, it shows that using subword information improves the tagging significantly. However, the subword information in noisy text does not need to be syntactic (morphological units) as suggested and character n-gram level features help in tagging substantially.

As for the date label, the week days can be tagged correctly. However, analogously if they span over multiple words, they cannot be identified.

A list of examples to errors in our Turkish results is given in Table 11.

\subsection{Experimental Results on English}

We performed a similar set of experiments by combining various word representations to measure the effect of different word and subword representation levels for the English noisy text. Analogously, we employed word based word embedding method word2vec (Mikolov et al., 2013), character n-gram level word embedding method fasttext (Bojanowski et al., 2017), morpheme level word embedding method morph2vec (Üstün and Can, 2016), character embeddings trained with a Bi-LSTM (and $\mathrm{CNN}$ ), and orthographic character-level embeddings trained on a character level Bi-LSTM. The overview of the English results are given in Table 12 and Table 13 for the baseline and the transfer learning models respectively.

Amongst using solely character-level embeddings, morph2vec (Üstün and Can, 2016), fasttext (Bojanowski et al., 2017), or word2vec (Mikolov et al., 2013), the 


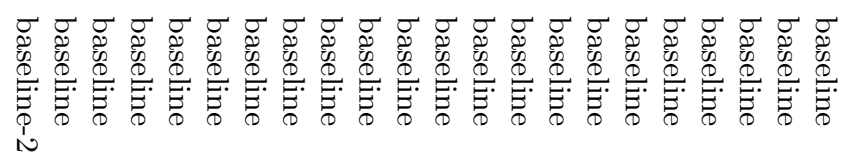

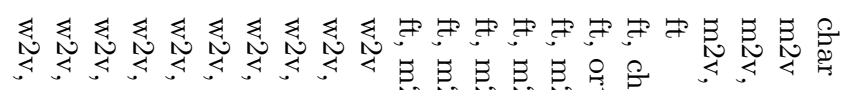

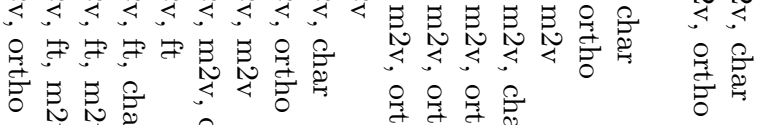

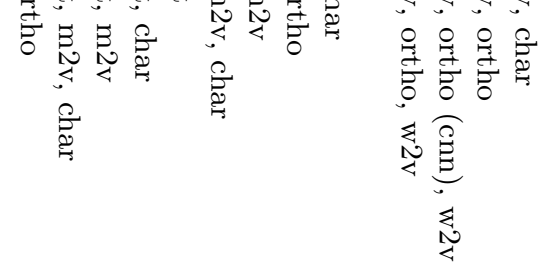

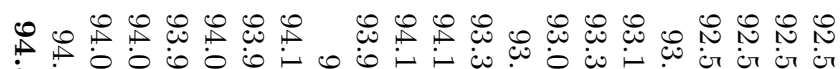

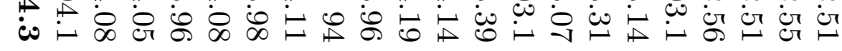

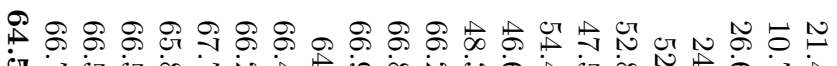

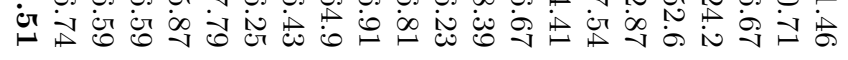

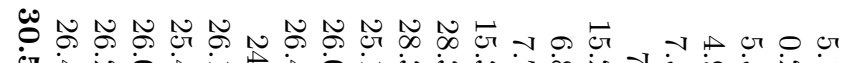

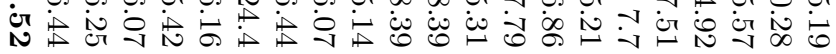

A w v

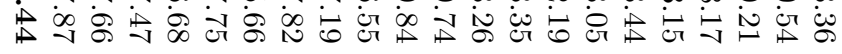

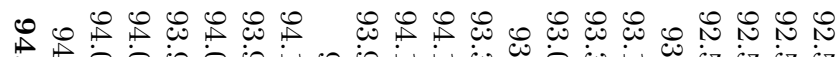

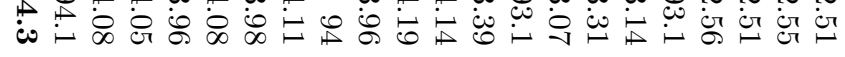

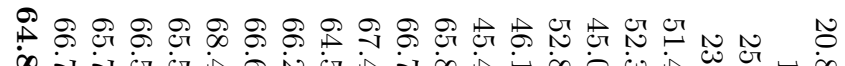

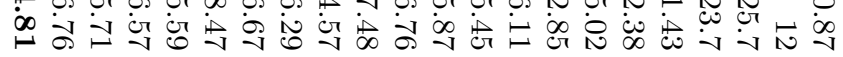

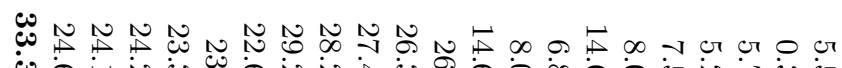

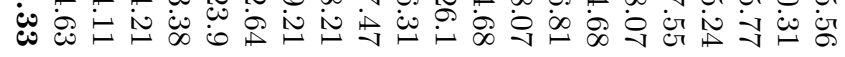

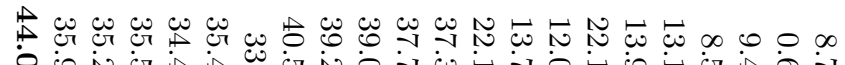

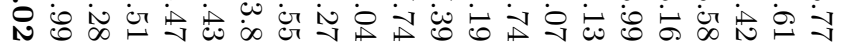

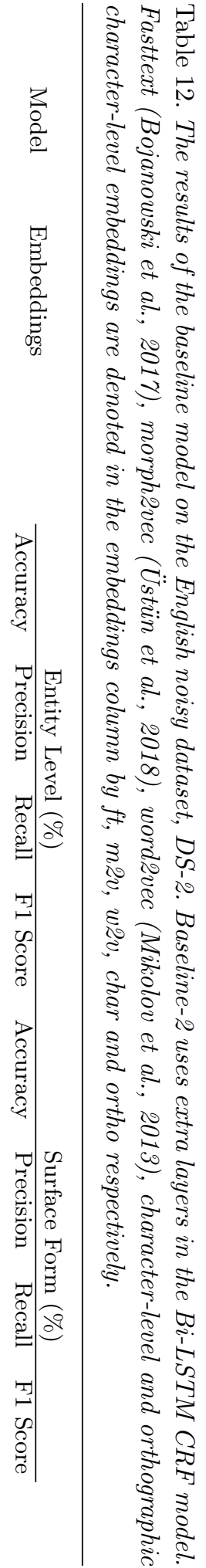




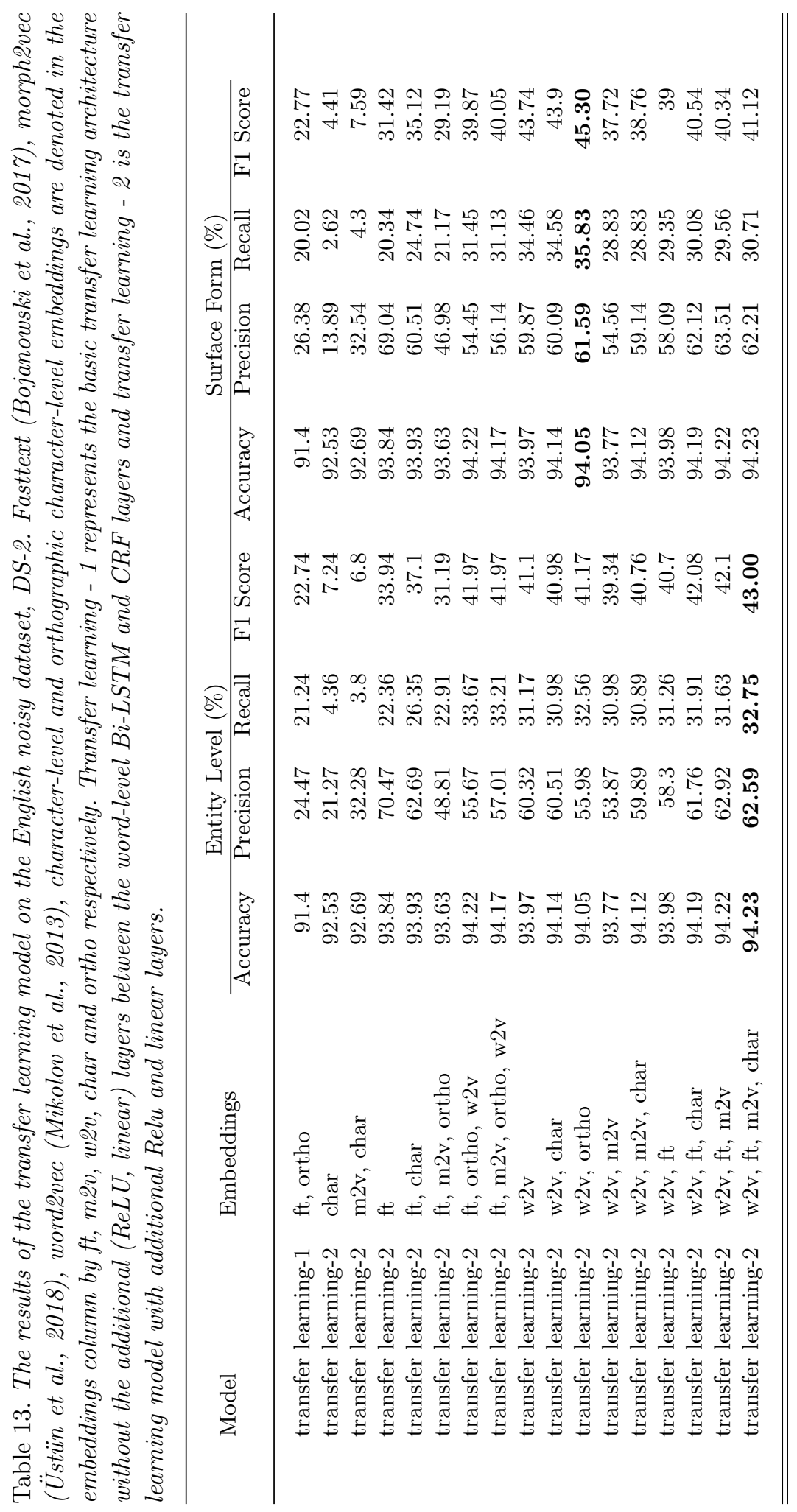


highest results are obtained from word2vec (Mikolov et al., 2013) with a F1 measure of $39.04 \%$ in the surface level and F1 measure of $36.55 \%$ in the entity level, which gives a completely different picture from the Turkish results where the highest score was obtained from fasttext with a F1 measure of 58.91\%. The English results are both lower than that of Turkish, and moreoever word-level embeddings are more beneficial in English compared to Turkish. Due to the morphological divergence between the two languages, obtaining a better performance from word-level word embeddings is an expected result. However, the performance is still not satisfactory compared to the highest result in Turkish when using a single type of word embedding.

Using orthographic character-level word embeddings in addition to word2vec contributed the most with a F1 measure of $40.55 \%$ in the surface level and $37.82 \%$ in the entity level. Although the other embedding types do not contribute on top of the word2vec embeddings in the surface level, character-level word embeddings, orthographic embeddings and fasttext embeddings slightly contribute to the word-level word embeddings, however the contribution is not more than $0.6 \%$.

Combining word2vec embeddings with other embeddings obtained from different levels still do not change the results and the highest results in the surface level still remains the same as the one obtained from using solely word2vec embeddings. However, in the entity level, the highest performance is obtained by using word2vec, fasttext, morph2vec, and orthographic word embeddings, which gives a F1 measure of $39.84 \%$. This is around $3 \%$ higher than the results obtained from using solely word2vec. However, in the surface level, the highest results are obtained by using word2vec and orthographic character embeddings with an F1 score of 40.55\%.

Without using any word-level word embeddings, the results are far behind the highest obtained score in English, and most of them are below 20\%. This concludes that word-level word embeddings of a morphologically poor language such as English bears further information compared to other embedding types and the other levels of word embeddings hardly contribute on top of the word-level word embeddings.

Here, orthographic character-level embeddings that are trained on CNN instead of Bi-LSTM performed poorer, thus we used only Bi-LSTM trained character-level word embeddings in all experiments on English.

The highest results obtained from different entity types are given in Table 14. We obtain the highest scores again for the most frequent entity types such as person and location, whereas the other sparse entity types such as corporation, product, creative-work or group cannot be detected as accurate as the frequent types.

In both transfer learning models, we used the English noisy dataset released by the 2 nd Workshop on Noisy User-generated Text workshop, WNUT' $16^{12}$ as a source dataset. Therefore, both source and target datasets are noisy but sizes of the datasets are different. The first transfer learning model, transfer learning - 1 has not improved upon the baseline and the results are even worse for this model.

12 https://noisy-text.github.io/2016/ 
Table 14. The results of the baseline model with word2vec (Mikolov et al., 2013), and orthographic character-level embeddings on English noisy dataset, DS-2

\begin{tabular}{lrrrrrr}
\hline \hline \multirow{2}{*}{ Entity Type } & \multicolumn{3}{c}{ Entity Level (\%) } & \multicolumn{2}{c}{ Surface Form $(\%)$} \\
\cline { 2 - 7 } & Precision & Recall & F1 score & Precision & Recall & F1 score \\
\hline corporation & 29.41 & 15.15 & 20.00 & 28.00 & 14.89 & 19.44 \\
creative-work & 53.85 & 4.93 & 9.07 & 53.85 & 5.83 & 10.53 \\
group & 48.72 & 11.52 & 18.63 & 47.06 & 12.6 & 19.88 \\
location & 69.57 & 42.67 & 52.89 & 69.01 & 40.83 & 51.31 \\
person & 74.79 & 41.59 & 53.45 & 75.25 & 53.41 & 62.47 \\
product & 53.85 & 5.51 & 10.00 & 50.00 & 5.56 & 10.00 \\
\hline overall & $\mathbf{6 6 . 4 3}$ & $\mathbf{2 6 . 4 4}$ & $\mathbf{3 7 . 8 2}$ & $\mathbf{6 6 . 2 9}$ & $\mathbf{2 9 . 2 1}$ & $\mathbf{4 0 . 5 5}$ \\
\hline \hline
\end{tabular}

We obtained an F1 score of $22.77 \%$ for the entity level and $22.74 \%$ for the surface level from transfer learning - 1 by using fasttext embeddings and orthographic character-level word embeddings.

As for the transfer learning model with additional layers, the results are significantly improved upon the baseline model accordingly. For example, using solely word2vec embeddings in the baseline model gives a F1 measure of 39.04\%, whereas it improves up to $43.74 \%$ in the surface level. The highest score is obtained with a F1 measure of $45.3 \%$ when orthographic embeddings are combined with the word2vec embeddings, which was also the highest in the baseline model. Likewise, fasttext embeddings do not perform well on the transfer learning model for English. Therefore, the results obtained from baseline model transfer learning model for different levels of embeddings are coherent with each other.

We also performed another experiment with the baseline model with additional layers similar to Turkish, which is called baseline-2 in Table 12. We used only word2vec and orthographic character embeddings in this setting since it gives the highest score in the surface level for the baseline model without the additional layers. Using the additional layers improves the F1 score up to $44.02 \%$, which is higher than F1 score of $40.55 \%$ obtained from the baseline model without the additional layers using the same embeddings. In Turkish, using additional layers in the baseline model does not help, whereas in English the additional layers contribute significantly.

Table 15 presents the highest obtained results for different entity types for the transfer learning model. It is clearly seen that transfer learning helps the model to learn rarely-seen entity types better compared to the baseline model, thus the overall results on both entity-level and surface forms are significantly improved. 
Table 15. Experimental results of transfer learning model (transfer learning - 2) with word2vec (Mikolov et al., 2013) and ortographic character-level embeddings on English noisy dataset, $D S$-2

\begin{tabular}{lrrrrrr}
\hline \hline \multirow{2}{*}{ Entity Type } & \multicolumn{3}{c}{ Entity Level (\%) } & \multicolumn{3}{c}{ Surface Form (\%) } \\
\cline { 2 - 7 } & Precision & Recall & F1 score & Precision & Recall & F1 score \\
\hline corporation & 37.84 & 21.21 & 27.18 & 40.00 & 21.28 & 27.78 \\
creative-work & 41.67 & 7.04 & 12.05 & 43.48 & 8.33 & 13.99 \\
group & 52.73 & 17.58 & 26.36 & 50.00 & 18.90 & 27.43 \\
location & 42.78 & 53.33 & 47.48 & 57.27 & 52.5 & 54.78 \\
person & 69.33 & 48.60 & 57.14 & 72.15 & 61.29 & 66.28 \\
product & 41.67 & 7.87 & 13.25 & 39.13 & 8.33 & 13.74 \\
\hline \multirow{2}{*}{ overall } & $\mathbf{5 5 . 9 8}$ & $\mathbf{3 2 . 5 6}$ & $\mathbf{4 1 . 1 7}$ & $\mathbf{6 1 . 5 9}$ & $\mathbf{3 5 . 8 3}$ & \multirow{4}{*}{$\mathbf{4 5 . 3 0}$} \\
\hline \hline
\end{tabular}

\subsubsection{Comparison with Related Work on English}

We present a comparison of our proposed models to the related work on English noisy dataset $D S$-2. The results are given in Table 16. Our transfer learning model with additional layers achieves competitive results for the entity level, whereas our baseline model and the transfer learning model with additional layers outperform all other models including the highest scoring models competed in WNUT'17, that are proposed by von Däniken and Cieliebak (2017) and Aguilar et al. (2017). The highest score in related work was achieved by Aguilar et al. (2017) with 40.24\% F1 score. Our transfer learning model gives $45.30 \% \mathrm{~F} 1$ score for the surface forms using the word2vec and orthographic character-level embeddings. However, applying McNemar test ${ }^{13}$ (McNemar, 1947) between the model proposed by Aguilar et al. (2017) and our transfer learning-2 model does not strongly imply this difference $(\mathrm{p}=0.248)$ in the surface level. If we compare the transfer learning- 2 model with the transfer learning model proposed by von Däniken and Cieliebak (2017) in the surface level, McNemar test confirms the significance of this difference, $p<0.05$. Therefore, our transfer learning-2 model significantly outperforms the transfer learning model of von Däniken and Cieliebak (2017) in the surface level. Additionally, our baseline model with additional layers (baseline-2) gives $41.44 \%$ F1 score for the entity level, which is competitive to that of Aguilar et al. (2017), where their highest reported result is $41.86 \%$ for the entity level. However, McNemar test between the baseline-2 and the model of Aguilar et al. (2017) shows that this difference is not significant in the entity level $(p=1.0)$. The same also applies for the difference between the transfer learning model of von Däniken and Cieliebak (2017) and baseline-2. On

${ }^{13}$ In particular, we applied McNemar-Bowker test that allows multiple categories in the results, whereas the original version of McNemar test allows only binary categories. 
Table 16. Comparison with related work on English noisy dataset DS-2. All results are comparable with each other.

\begin{tabular}{lcc}
\hline \hline \multirow{2}{*}{ Related Work } & \multicolumn{2}{c}{ F1 score (\%) } \\
\cline { 2 - 3 } & Entity Level & Surface Form \\
\hline Jansson and Liu (2017) & 39.98 & 37.77 \\
Williams and Santia (2017) & 26.30 & 25.26 \\
Sikdar and Gambäck (2017) & 38.35 & 36.31 \\
Lin et al. (2017) & 40.42 & 37.62 \\
von Däniken and Cieliebak (2017) & 40.78 & 39.33 \\
Aguilar et al. (2017) & $\mathbf{4 1 . 8 6}$ & 40.24 \\
\hline baseline (w2v, ortho) & 37.82 & 40.55 \\
baseline-2 (w2v, ortho) & 41.44 & 44.02 \\
transfer learning-1 (w2v, ortho) & 22.74 & 22.77 \\
transfer learning-2 (w2v, ortho) & 41.17 & $\mathbf{4 5 . 3 0}$ \\
\hline \hline
\end{tabular}

the other hand, McNemar test shows that the difference between baseline-2 model and the model proposed by Lin et al. (2017) is significant $(p<0.05)$.

It should be noted that both Aguilar et al. (2017) and von Däniken and Cieliebak (2017) make use of hand-crafted features such as capitalization or domain-specific knowledge such as gazetteers, whereas our models do not use any external resource.

\subsubsection{Error Analysis}

Since English is not a morphologically rich language, the errors do not occur because of the inflection of the named entities. Instead, most of the errors are due to the sparsity in the data. The variety of the proper names (i.e., word types) in English data is quite intense compared to Turkish data. Hence, the infrequent named entities cannot be identified analogously to Turkish. For example, although the name 'Thomas Jane' is correctly tagged as person, 'Groep Klein' cannot be tagged correctly as person since it is not as frequent as the former.

Analogously, location, organization, and person names that span across multiple words and that are also infrequent cannot be tagged correctly. However, the frequent named entities with multiple words can be tagged correctly (e.g. 'Fly Community Theater'). The English text is over-capitalized compared to Turkish text and even the common names could be capitalized. This leads to mistagging especially in location names. For example, 'Hotel Housekeepers Needed' is incorrectly tagged as location because of the location word 'hotel' that is capitalized. On the other hand, 'newzealand' is tagged as other, since the word embeddings do not help in those multiword entities.

Corporation names are usually tagged as group. Moreover, product names and 
Table 17. A list of incorrect tags in English

\begin{tabular}{|c|c|}
\hline & Examples \\
\hline $\begin{array}{l}\text { Predicted } \\
\text { Correct }\end{array}$ & $\begin{array}{lr}\text { Living } \backslash O \text { Computer } \backslash \mathrm{O} \text { Museum } \backslash \mathrm{O} & \\
\text { Living } \backslash \text { B-LOCATION } \quad \text { Computer } \backslash \text { I-LOCATION } & \text { Museum } \backslash \text { I- } \\
\text { LOCATION } & \end{array}$ \\
\hline $\begin{array}{l}\text { Predicted } \\
\text { Correct }\end{array}$ & $\begin{array}{l}\text { Groep } \backslash O \text { Klein } \backslash O \\
\text { Groep } \backslash \text { B-PERSON Klein } \backslash \text { I-PERSON }\end{array}$ \\
\hline $\begin{array}{l}\text { Predicted } \\
\text { Correct }\end{array}$ & $\begin{array}{l}\text { Supreme } \backslash O \text { Court } \backslash O \text { judge } \backslash O \\
\text { Supreme } \backslash \text { B-PERSON Court } \backslash \text { I-PERSON judge } \backslash I-P E R S O N\end{array}$ \\
\hline $\begin{array}{l}\text { Predicted } \\
\text { Correct }\end{array}$ & $\begin{array}{lr}\text { Great } \backslash \text { O Southern } \backslash \text { B-GROUP Television } \backslash \text { I-GROUP } \\
\text { Great } \backslash \text { B-CORPORATION } & \text { Southern } \backslash \text { I-CORPORATION } \\
\text { Television } \backslash \text { I-CORPORATION } & \end{array}$ \\
\hline $\begin{array}{l}\text { Predicted } \\
\text { Correct }\end{array}$ & $\begin{array}{l}\text { Snickers } \backslash \text { B-CORPORATION } \\
\text { Snickers } \backslash \text { B-PRODUCT }\end{array}$ \\
\hline $\begin{array}{l}\text { Predicted } \\
\text { Correct }\end{array}$ & $\begin{array}{l}\text { Zealandia } \backslash \mathrm{O} \\
\text { Zealandia } \backslash \mathrm{B}-\mathrm{LOCATION}\end{array}$ \\
\hline $\begin{array}{l}\text { Predicted } \\
\text { Correct }\end{array}$ & $\begin{array}{l}\text { Amazon } \backslash \text { B-CORPORATION Echo } \backslash \mathrm{O} \\
\text { Amazon } \backslash \text { B-PRODUCT Echo } \backslash \text { I-PRODUCT }\end{array}$ \\
\hline $\begin{array}{l}\text { Predicted } \\
\text { Correct }\end{array}$ & $\begin{array}{l}\text { Turkish } \backslash O \text { military } \backslash O \\
\text { Turkish } \backslash \text { B-GROUP military } \backslash \text { I-GROUP }\end{array}$ \\
\hline $\begin{array}{l}\text { Predicted } \\
\text { Correct }\end{array}$ & 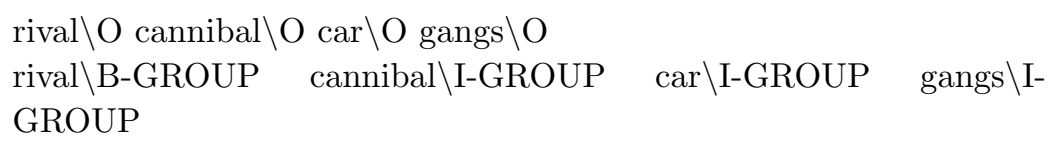 \\
\hline
\end{tabular}

creative work are tagged as corporation in general. Since the number of those entities are not $\mathrm{su}$ cient to be learned in the noisy text, they cannot be identified properly. For example, corporation names are identified correctly, if they are frequent (e.g. 'reddit'). Most of the time, the group names cannot be identified and tagged as other. The same also applies for the product names. For example, 'Chevrolet Corvette' is tagged correctly as product, whereas 'Centrelink' and 'Sudocrem' are tagged as other.

A list of examples to errors in our English results is given in Table 17. 


\section{Conclusion \& Future Work}

Various attempts have been made on Turkish named entity recognition recently. However, the results are still not satisfactory for noisy text. In this article, we have investigated using deep neural networks along with transfer learning instead of using rule-based or statistical approaches for named entity recognition on noisy Turkish text. Noisy text has its own di culties because of the very sparse orthography of words that highly depend on the user style. Moreover, Turkish brings more challenges due to its morphologically rich structure, which introduces more sparsity in the text. We have investigated the effects of using different word and sub-word level word representation methods such as word-level, character n-gram level, morpheme-level, and orthographic character-level embeddings to mitigate the sparsity in text. We did not use any hand-crafted features and external resources unlike the other existing studies on Turkish named entity recognition on noisy text. We investigated transfer learning between a formal text and a noisy (informal) text in order to deal with the sparsity issue in the noisy text.

Therefore, we obtained the highest scores for Turkish named entity recognition on noisy text by using a combination of word-level and sub-word embeddings with transfer learning between a formal text and noisy text. We have also experimented with English as a relatively morphologically-poor language and obtained the highest surface-level score and competitive entity-level scores on the English noisy dataset.

The results show that sub-word information plays a vital role for the named entity recognition task, especially on morphologically-rich languages. More importantly, we can successfully learn valuable information without using hand-crafted features or domain-specific external resources. Furthermore, it is also proven that transfer learning approach can indeed effectively be used to tackle the problem of data scarcity.

Since our model is not domain and language specific, we believe it can also be effectively trained and used for other morphologically-rich languages, especially those with data sparsity problem. Therefore, experimenting for different languages remains as a future goal.

\section{References}

Aguilar, G., Maharjan, S., Monroy, A. P. L., and Solorio, T. 2017. A multitask approach for named entity recognition in social media data. In Proceedings of the 3rd Workshop on Noisy User-generated Text, pp. 148-153.

Bikel, D. M., Miller, S., Schwartz, R., and Weischedel, R. 1997. Nymble: A high-performance learning name-finder. In Proceedings of the Fifth Conference on Applied Natural Language Processing, ANLC '97, pp. 194-201, Stroudsburg, PA, USA. Association for Computational Linguistics.

Blei, D. M., Ng, A. Y., and Jordan, M. I. 2003. Latent Dirichlet allocation. Journal of Machine Learning Research, 3:993-1022.

Bojanowski, P., Grave, E., Joulin, A., and Mikolov, T. 2017. Enriching word vectors with subword information. Transactions of the Association for Computational Linguistics, 5:135-146. 
Cao, K. and Rei, M. 2016. A joint model for word embedding and word morphology. In Proceedings of the 1st Workshop on Representation Learning for NLP, pp. 18-26. Association for Computational Linguistics.

Çelikkaya, G., Torunoğlu, D., and Eryiğit, G. 2013. Named entity recognition on real data: a preliminary investigation for Turkish. In Application of Information and Communication Technologies (AICT), 2013 7th International Conference on, pp. 1-5. IEEE.

Chiu, J. P. and Nichols, E. 2015. Named entity recognition with bidirectional LSTM-CNNs. arXiv preprint arXiv:1511.08308.

Cotterell, R. and Duh, K. 2017. Low-resource named entity recognition with cross-lingual, character-level neural conditional random fields. In Proceedings of the Eighth International Joint Conference on Natural Language Processing (Volume 2: Short Papers), pp. 91-96, Taipei, Taiwan. Asian Federation of Natural Language Processing.

Derczynski, L., Nichols, E., van Erp, M., and Limsopatham, N. 2017. Results of the WNUT2017 shared task on novel and emerging entity recognition. In Proceedings of the 3rd Workshop on Noisy User-generated Text, pp. 140-147.

Eken, B. and Tantuğ, C. 2015. Recognizing named entities in Turkish tweets. In Proceedings of the Fourth International Conference on Software Engineering and Applications, Dubai, UAE.

Glorot, X. and Bengio, Y. 2010. Understanding the di culty of training deep feedforward neural networks. In Teh, Y. W. and Titterington, M., editors, Proceedings of the Thirteenth International Conference on Artificial Intelligence and Statistics, volume 9 of Proceedings of Machine Learning Research, pp. 249256, Chia Laguna Resort, Sardinia, Italy. PMLR.

Godin, F., Vandersmissen, B., De Neve, W., and Van de Walle, R. 2015. Multimedia lab @ ACL WNUT NER shared task: Named entity recognition for Twitter microposts using distributed word representations. In Proceedings of the Workshop on Noisy User-generated Text, pp. 146-153.

Hochreiter, S. and Schmidhuber, J. 1997. Long short-term memory. Neural computation, 9(8):1735-1780.

Huang, Z., Xu, W., and Yu, K. 2015. Bidirectional LSTM-CRF models for sequence tagging. arXiv preprint arXiv:1508.01991.

Jansson, P. and Liu, S. 2017. Distributed representation, LDA topic modelling and deep learning for emerging named entity recognition from social media. In Proceedings of the 3rd Workshop on Noisy User-generated Text, pp. 154-159.

Küçük, D. and Steinberger, R. 2014. Experiments to improve named entity recognition on Turkish tweets. arXiv preprint arXiv:1410.8668.

Lample, G., Ballesteros, M., Subramanian, S., Kawakami, K., and Dyer, C. 2016. Neural architectures for named entity recognition. arXiv preprint arXiv:1603.01360.

Landauer, T. K., Foltz, P. W., and Laham, D. 1998. An introduction to latent semantic analysis. Discourse Processes, 25(2-3):259-284.

Limsopatham, N. and Collier, N. H. 2016. Bidirectional LSTM for named entity recognition in Twitter messages. COLING 2016. 
Lin, B. Y., Xu, F., Luo, Z., and Zhu, K. 2017. Multi-channel BiLSTM-CRF model for emerging named entity recognition in social media. In Proceedings of the 3rd Workshop on Noisy User-generated Text, pp. 160-165.

Ma, X. and Hovy, E. 2016. End-to-end sequence labeling via bi-directional LSTM-CNNs-CRF. arXiv preprint arXiv:1603.01354.

McNemar, Q. 1947. Note on the sampling error of the difference between correlated proportions or percentages. Psychometrika, 12(2):153-157.

Mikolov, T., Chen, K., Corrado, G., and Dean, J. 2013. E cient estimation of word representations in vector space. arXiv preprint arXiv:1301.3781.

Nair, V. and Hinton, G. E. 2010. Rectified linear units improve restricted Boltzmann machines. In Proceedings of the 27th International Conference on International Conference on Machine Learning, ICML'10, pp. 807-814, USA. Omnipress.

Okur, E., Demir, H., and Özgür, A. 2016. Named entity recognition on Twitter for Turkish using semi-supervised learning with word embeddings. In LREC.

Pagliardini, M., Gupta, P., and Jaggi, M. 2017. Unsupervised learning of sentence embeddings using compositional n-gram features. arXiv preprint arXiv:1703.0250\%.

Pennington, J., Socher, R., and Manning, C. D. 2014. Glove: Global vectors for word representation. In Empirical Methods in Natural Language Processing (EMNLP), pp. 1532-1543.

Petasis, G., Vichot, F., Wolinski, F., Paliouras, G., Karkaletsis, V., and Spyropoulos, C. D. 2001. Using machine learning to maintain rule-based named-entity recognition and classification systems. In Proceedings of the 39th Annual Meeting on Association for Computational Linguistics, ACL '01, pp. 426433, Stroudsburg, PA, USA. Association for Computational Linguistics.

Reimers, N., Eckle-Kohler, J., Schnober, C., Kim, J., and Gurevych, I. 2014. Germeval-2014: Nested named entity recognition with neural networks.

Riedl, M. and Padó, S. 2018. A named entity recognition shootout for German. In Proceedings of the 56th Annual Meeting of the Association for Computational Linguistics (Volume 2: Short Papers), pp. 120-125, Melbourne, Australia. Association for Computational Linguistics.

Sak, H., Güngör, T., and Saraçlar, M. 2008. Turkish language resources: Morphological parser, morphological disambiguator and web corpus. In Advances in natural language processing, pp. 417-427. Springer.

Sak, H., Güngör, T., and Saraçlar, M. 2011. Resources for Turkish morphological processing. Language resources and evaluation, 45(2):249-261.

Şeker, G. A. and Eryiğit, G. 2017. Extending a CRF-based named entity recognition model for Turkish well formed text and user generated content 1. Semantic Web, 8(5):625-642.

Sezer, B., Sezer, T., and Ünivesitesi, M. 2013. TS Corpus: Herkes için Türkçe derlem. In Proceedings 27th National Linguistics Conference. May, pp. 3-4.

Sikdar, U. K. and Gambäck, B. 2017. A feature-based ensemble approach to recognition of emerging and rare named entities. In Proceedings of the 3rd Workshop on Noisy User-generated Text, pp. 177-181. 
Suzuki, J. and Isozaki, H. 2008. Semi-supervised sequential labeling and segmentation using giga-word scale unlabeled data. In Proceedings of ACL-08: HLT, pp. 665-673. Association for Computational Linguistics.

Torunoğlu, D. and Eryiğit, G. 2014. A cascaded approach for social media text normalization of Turkish. In Proceedings of the 5th Workshop on Language Analysis for Social Media (LASM), pp. 62-70.

Tür, G., Hakkani-Tür, D., and Oflazer, K. 2003. A statistical information extraction system for Turkish. Natural Language Engineering, 9(2):181-210.

Üstün, A. and Can, B. 2016. Unsupervised morphological segmentation using neural word embeddings. In Král, P. and Martín-Vide, C., editors, Statistical Language and Speech Processing, pp. 43-53, Cham. Springer International Publishing.

Üstün, A., Kurfalı, M., and Can, B. 2018. Characters or morphemes: How to represent words? In Proceedings of The Third Workshop on Representation Learning for NLP, pp. 144-153.

von Däniken, P. and Cieliebak, M. 2017. Transfer learning and sentence level features for named entity recognition on tweets. In Proceedings of the 3rd Workshop on Noisy User-generated Text, pp. 166-171.

Williams, J. and Santia, G. 2017. Context-sensitive recognition for emerging and rare entities. In Proceedings of the 3rd Workshop on Noisy User-generated Text, pp. 172-176.

Wu, Y., Zhao, J., and Xu, B. 2003. Chinese named entity recognition combining statistical model with human knowledge. In Proceedings of the ACL 2003 Workshop on Multilingual and Mixed-language Named Entity Recognition.

Yang, Z., Salakhutdinov, R., and Cohen, W. W. 2017. Transfer learning for sequence tagging with hierarchical recurrent networks. arXiv preprint arXiv:1703.06345.

Yin, Z. and Shen, Y. 2018. On the dimensionality of word embedding. In Proceedings of the 32Nd International Conference on Neural Information Processing Systems, NIPS'18, pp. 895-906, USA. Curran Associates Inc. 